\title{
Job Demands and Worker Health: Three-Dimensional Reexamination of the Relationship Between Person-Environment Fit and Strain
}

\author{
Jeffrey R. Edwards and R. Van Harrison
}

\begin{abstract}
The most influential study of the person-environment (P-E) fit approach to stress was conducted by J. R. P. French, R. D. Caplan, and R. V. Harrison (1982). Unfortunately, this study operationalized fit using various transformations of difference scores, thereby introducing numerous substantive and methodological problems. In the present study, the authors reanalyze data from French et al., using a procedure described by $J$. R. Edwards (in press) that avoids problems with difference scores and captures the underlying three-dimensional relationship between $E, P$, and strain. Results resolve ambiguities in the French et al. findings and identify relationships between $E$, $P$, and strain that, although consistent with P-E fit theory, cannot be adequately represented by fit measures such as those used by French et al. Implications for P-E fit research are discussed.
\end{abstract}

The person-environment (P-E) fit approach to stress (Caplan, Cobb, French, Harrison, \& Pinneau, 1980; French, Caplan, \& Harrison, 1982) has become widely accepted among organizational stress researchers (Eulberg, Weekley, \& Bhagat, 1988). In essence, the P-E fit approach states that misfit between the person and the environment may produce psychological, physiological, and behavioral strains (e.g., dissatisfaction, elevated serum cholesterol, and smoking) that ultimately increase morbidity and mortality. These basic principles underlie numerous theories of stress in organizations, such as those proposed by McGrath (1976), Karasek (1979), Schuler (1980), Beehr and Bhagat (1985), and Edwards (1992).

The most comprehensive study of P-E fit to date was conducted by French et al. (1982; see also Caplan et al., 1980). This study measured $E$ and $P$ variables on eight job dimensions, used five operationalizations of fit, and included 18 measures of psychological and physiological strain. The results of this study yielded three major conclusions. First, misfit was frequently associated with increased strain, particularly job-related affect and psychological disturbance. Second, the relationship between misfit and strain was often curvilinear, with a turning point where $E$ and $P$ were equal. Third, fit measures representing these curvilinear relationships often accounted for significant variance beyond that explained by $E$ and $P$ mea-

Jeffrey R. Edwards, Darden Graduate School of Business Administration, University of Virginia; R. Van Harrison, Medical School and Institute for Social Research, University of Michigan.

Support for this research was provided in part by Contract HSM-9972-61 from the National Institute of Occupational Safety and Health, by the Institute for Social Research at the University of Michigan, and by the Darden Graduate School of Business Administration Foundation at the University of Virginia. We thank A. J. Baglioni, Jr., for his help in managing the data and Mark E. Parry, Shyamal Peddada, Don Richards, and Michael A. Seaman for their helpful comments during the preparation of this article.

Correspondence concerning this article should be addressed to Jeffrey R. Edwards, who is now at the School of Business Administration, University of Michigan, Ann Arbor, Michigan 48109-1234. sures, typically doubling the proportion of variance explained in strain.

Although the French et al. (1982) study is undoubtedly a landmark in the P-E fit literature, it operationalized fit using various transformations of the algebraic difference between $E$ and $P$. As is widely known, difference scores suffer from numerous substantive and methodological problems (Cronbach \& Furby, 1970; Edwards \& Cooper, 1990; Johns, 1981; Wall \& Payne, 1973; Werts \& Linn, 1970). In fairness, we acknowledge that French et al. conducted their study before many of these problems had been identified and that methods that overcome these problems have only recently been developed (Edwards, in press). Nonetheless, these problems severely limit the conclusiveness of what remains the most important P-E fit study to date.

Our purpose in this article was to reanalyze data from the French et al. (1982) study, using the procedure described by Edwards (in press). This procedure involves regressing strain on $E, P$, and certain higher order terms, such as the square of $E$ and $P$, their product, and so on, supplemented by tests that explicitly evaluate the conceptual models underlying the fit measures used by French et al. As we show, this procedure avoids problems with fit measures; clarifies and elaborates the relationship between $E, P$, and strain; and shows that the magnitude of this relationship was often significantly underestimated.

\section{Overview of P-E Fit Theory}

As indicated earlier, the central hypothesis of P-E fit theory is that misfit between the person and the environment leads to psychological, physiological, and behavioral strains, which ultimately increase morbidity and mortality (Caplan, 1987; French et al., 1982; Harrison, 1978, 1985). P-E fit can take either of two forms, one representing the extent to which the rewards and supplies provided by the environment match the needs and preferences of the person, and the other representing the extent to which the demands and requirements of the environment match the skills and abilities of the person. Strains encompass various deviations from normal functioning, such as dissatisfac- 
tion, boredom, anxiety, depression, hypertension, elevated serum cholesterol, smoking, and so on.

P-E fit theory proposes three basic hypothetical relationships between fit and strain. These relationships are embodied in the five fit measures used by French et al. (1982) and Caplan et al. (1980). The simplest of these measures, labeled fit, consists of the algebraic difference between $E$ and $P(E-P)$ and was intended to depict a monotonic relationship with strain (see Figure 1a). This relationship is expected when, for example, strain not only decreases as supplies increase toward motives but continues to decrease thereafter, as when excess supplies can be applied toward other motives or retained for future use. Two measures, labeled deficiency $(E-P$ for $E \leq P, 0$ for $E>P$ ) and excess $(E-P$ for $E \geq P, 0$ for $E<P$ ), were intended to depict asymptotic relationships with strain. Deficiency represents a negative relationship with strain only when $E$ is less than $P$ (Figure 1c), as when increasing supplies reduce strain up to the point of satiation but have little effect thereafter. In contrast, excess represents a positive relationship with strain only when $E$ is greater than $P$ (Figure le), as when demands increase strain when they exceed abilities but not when they fall short of abilities. Two measures, poor fit $(|E-P|)$ and the squared difference between $E$ and $P\left((E-P)^{2}\right.$, here labeled fit squared $)$, were intended to represent curvilinear relationships with strain (Figures $\mathrm{lg}$ and $\mathrm{li}$, respectively). These relationships are expected when either excess or inadequate supplies or demands are harmful, as when too much job complexity leads to overload but too little creates boredom. Although P-E fit theory clearly distinguishes these different relationships, it does not specifically predict which is more likely to occur in a given instance. Therefore, all five fit measures were used in the analyses reported in French et al. and Caplan et al.

\section{Problems With Fit Measures Used by French Et Al. (1982)}

Because the five fit measures used by French et al. (1982) were based on difference scores, they introduce numerous substantive and methodological problems (Cronbach \& Furby, 1970; Edwards, in press; Edwards \& Cooper, 1990; Johns, 1981; Wall \& Payne, 1973; Werts \& Linn, 1970). For example, these measures confound the separate relationships of $E$ and $P$ with strain. For some job dimensions, only $E$ or $P$ was significantly correlated with strain, suggesting that relationships for the corresponding fit measures may have reflected the influence of only one component. If so, then results for those fit measures are spurious (Wall \& Payne, 1973).

Second, fit measures with different substantive interpretations often yielded significant relationships with strain. For example, for job complexity, deficiency was negatively related to job dissatisfaction, indicating that dissatisfaction fell as $E$ increased toward $P$, but remained constant as $E$ exceeded $P$ (see Figure 1c). However, excess was also positively related to job dissatisfaction, indicating that dissatisfaction was constant as $E$ increased toward $P$, but increased thereafter (Figure $1 \mathrm{e}$ ). In addition, poor fit and fit squared were both positively related to job dissatisfaction (Figures $\mathrm{lg}$ and $\mathrm{li}$, respectively), indicating that dissatisfaction increased symmetrically as $E$ either exceeded or fell short of $P$. Taken together, these results suggest that the actual underlying relationship was probably some hybrid of those represented by the deficiency, excess, poor fit, and fit squared measures, but the exact nature of that relationship remains ambiguous. Significant correlations for more than one fit measure were also found for other job dimensions and indexes of strain, resulting in similar ambiguities (Caplan et al., 1980; French et al., 1982).

Third, fit measures effectively impose an untested set of constraints on the joint relationships of $E$ and $P$ with strain. For example, using fit to predict strain is equivalent to regressing strain on $E$ and $P$ with their coefficients constrained to be equal in magnitude but opposite in sign (Edwards, in press; Edwards \& Cooper, 1990). Deficiency, excess, poor fit, and fit squared impose additional constraints on the coefficients on $E$, $P$, and certain higher order terms these measures implicitly include, as detailed later in this article. Although these constraints were not tested by French et al. (1982), their results suggest that these constraints may be overly restrictive. For example, the relationships of $E$ and $P$ with strain often differed in absolute magnitude or were of the same sign, contrary to the constraint imposed by the fit measure. Similarly, relationships for deficiency and excess often differed in absolute magnitude, contrary to the symmetry implied by the poor fit and fit squared measures. Unfortunately, these comparisons are inconclusive without explicit tests of the constraints imposed by these measures. If these constraints are rejected, then the corresponding fit measures misrepresent the underlying relationship between $E, P$, and strain.

Finally, fit measures reduce the inherently three-dimensional relationship between $E, P$, and strain to two dimensions. This is illustrated in Figure 1, which compares hypothetical two-dimensional relationships between strain and the five fit measures used by French et al. (1982) to their three-dimensional counterparts. As Figure 1 shows, each fit measure embodies the effects of both $E$ and $P$ as joint predictors of strain. If $E$ and $P$ are indeed distinct constructs, as indicated by $P-E$ fit theory (Caplan, 1987; French et al., 1982; Harrison, 1978), then their relationship with strain is necessarily viewed in three dimensions, with $E$ and $P$ constituting two perpendicular horizontal axes and strain constituting the vertical axis. Collapsing this relationship into two dimensions violates the conceptual distinctions between $E$ and $P$, oversimplifies the potential complexity of their joint effects on strain, and prevents tests of certain effects suggested by P-E fit theory, such as variation in strain along the $E=P$ line (French et al., 1982; Harrison, 1978).

\section{Three-Dimensional Approach to Examining the Relationship Between P-E Fit and Strain}

Problems with the fit measures used by French et al. (1982) can be overcome by using the procedure described by Edwards (in press; see also Edwards, 1991; Edwards \& Cooper, 1990). This procedure involves regressing strain on $E, P$, and certain higher order terms, such as the square of $E$ and $P$, their product, and so on. Because $E$ and $P$ are used as separate predictors, their effects on strain are not confounded, and the three-dimensional relationship between $E, P$, and strain is preserved. Furthermore, when constraints on certain combinations of coef- 
a. Two-Dimensional Depiction of Fit

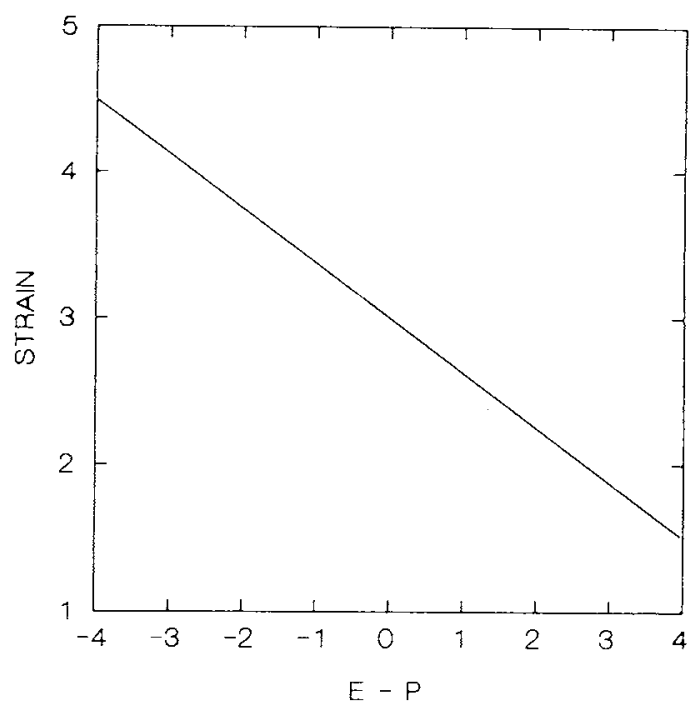

c. Two-Dimensional Depiction of Deficiency

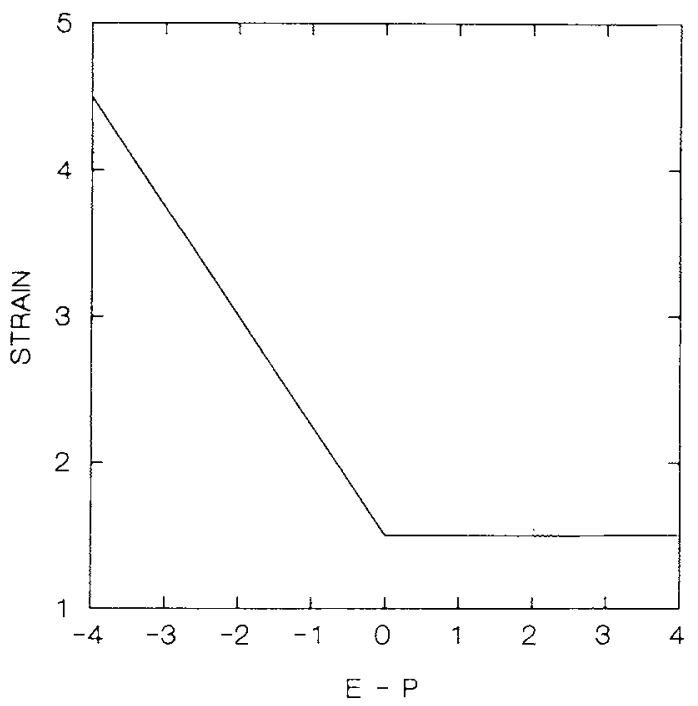

e. Two-Dimensional Depiction of Excess

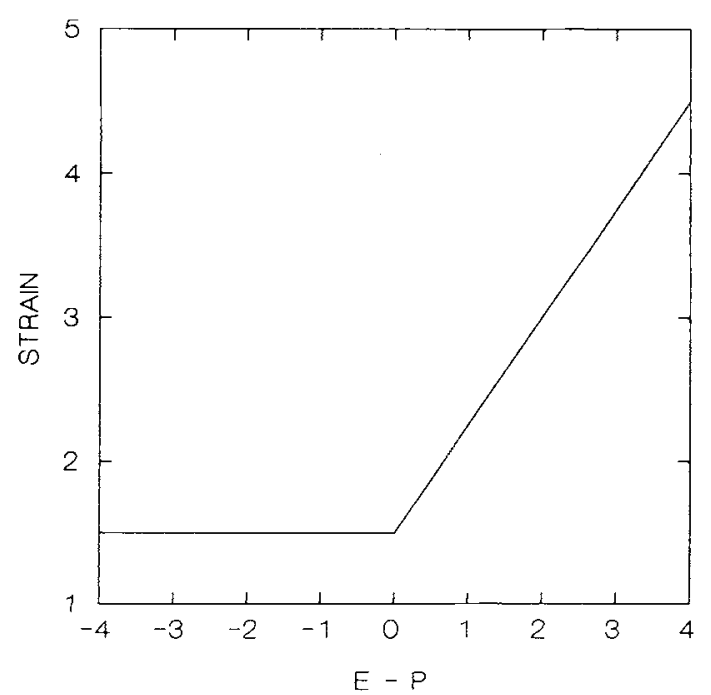

b. Three-Dimensional Depiction of Fit

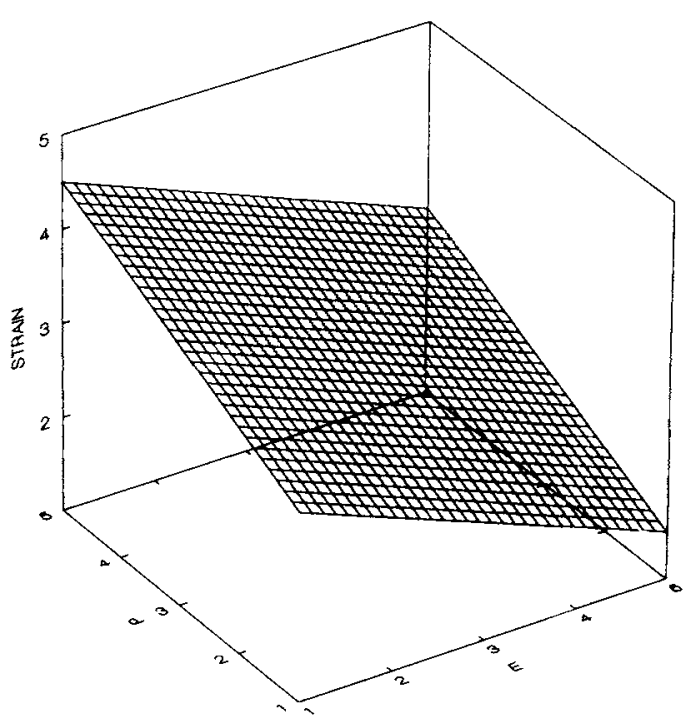

d. Three-Dimensional Depiction of Deficiency

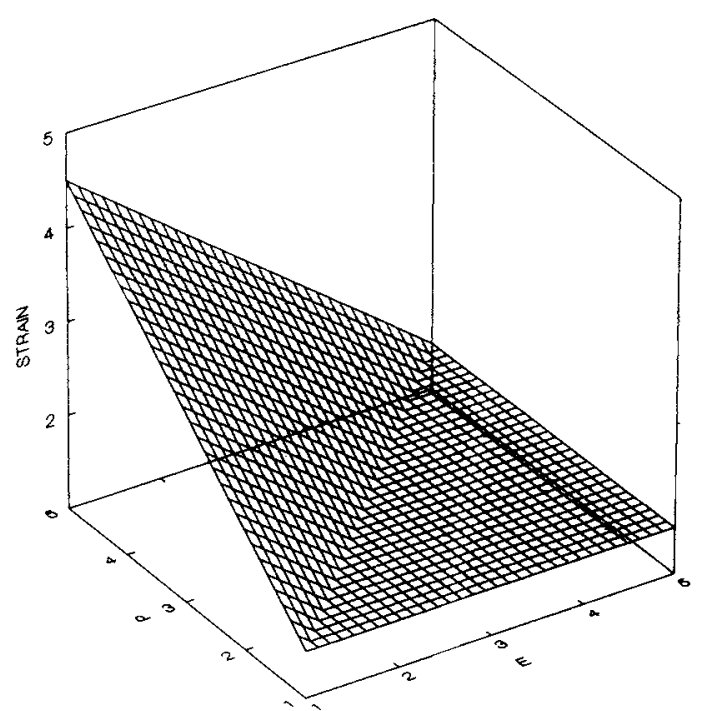

f. Three-Dimensional Depiction of Excess

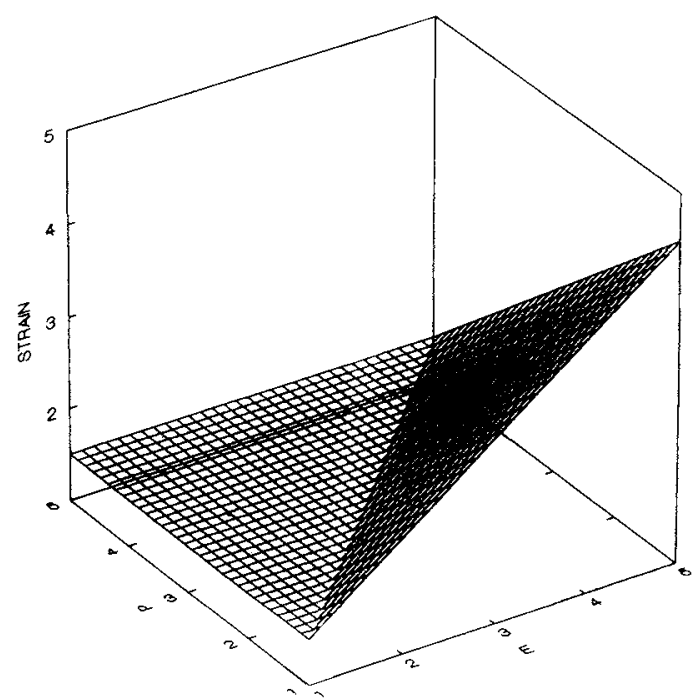


g. Two-Dimensional Depiction of Poor Fit

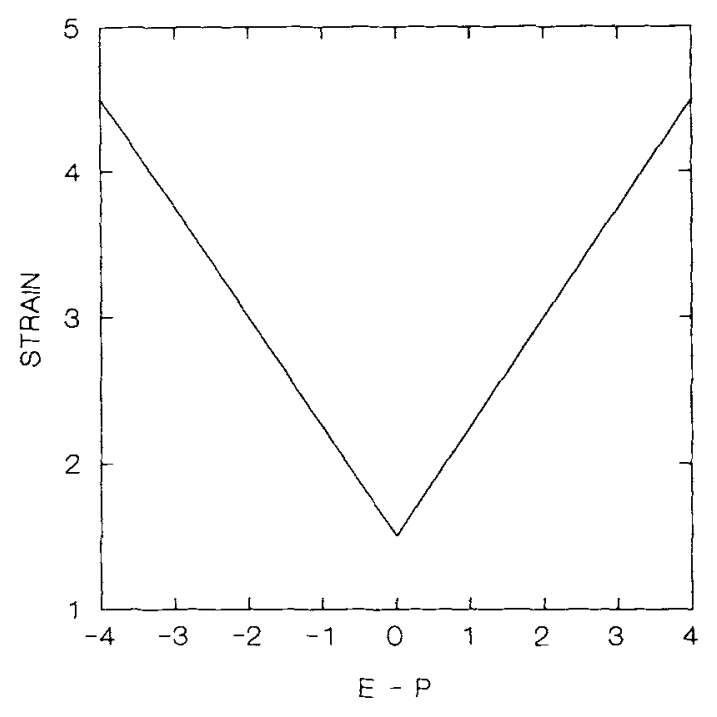

i. Two-Dimensional Depiction of Fit Squared

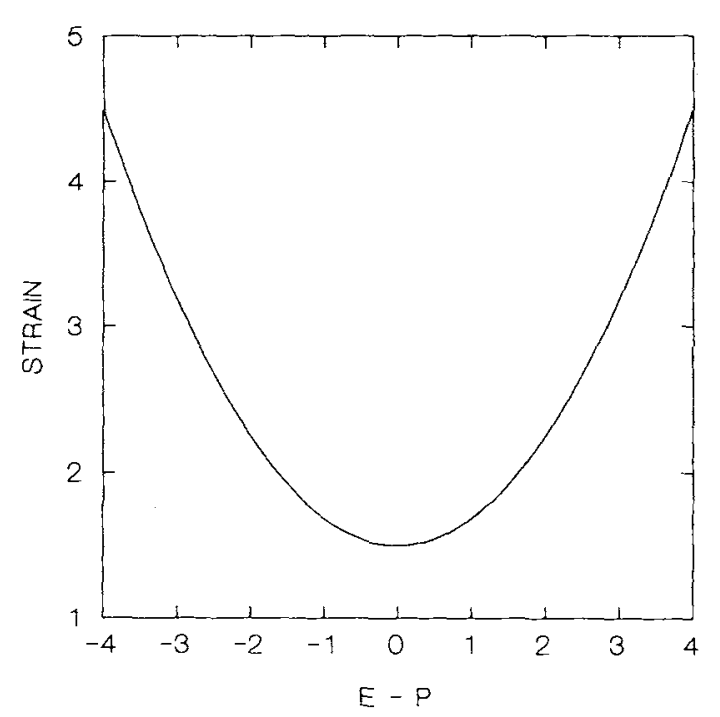

n. Three-Dimensional Depiction of Poor Fit

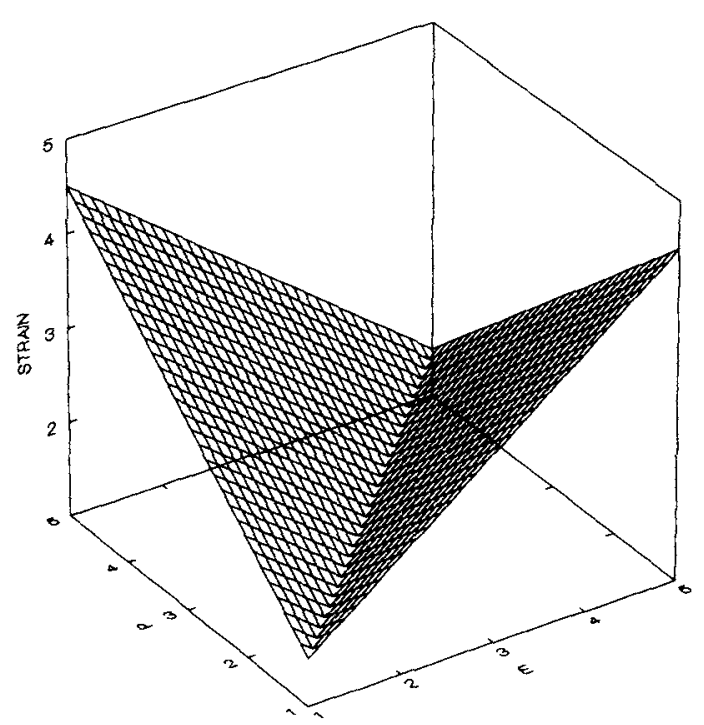

1. Three-Dimensional Depiction of Fit Squared

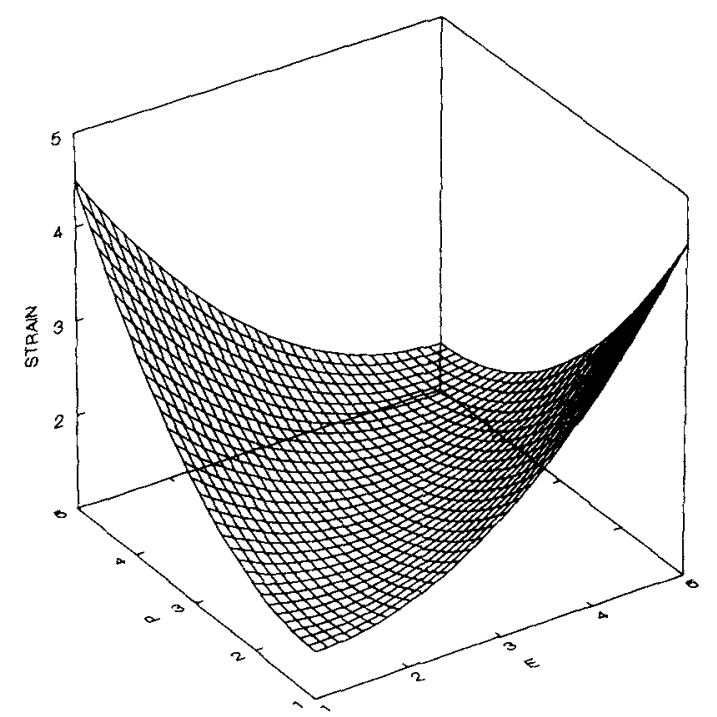

Figure 1 (left and above). Two- and three-dimensional depictions of relationships between personenvironment (P-E) fit measures and strain.

ficients are imposed, the regression equations become mathematically equivalent to fit measures such as those used by French et al., thereby allowing explicit tests of their associated constraints. In addition, surfaces more complex than those represented by fit measures can be analyzed, because the constraints imposed by these measures can be relaxed and higher order terms representing additional inflections and curvatures in the underlying surface can be included.

The benefits of this procedure have been demonstrated by Edwards (in press), who examined the relationship between satisfaction and fit across nine job dimensions, using data from 172 master of business administration students and 161 execu- tives. Measures representing fit, poor fit, fit squared, and various forms of profile similarity $\left(D^{2}, D,|D|\right.$, and $Q$; see Cronbach \& Gleser, 1953) were compared with regression equations that relaxed the constraints imposed by these measures. In all but one instance, the constraints were rejected. Three-dimensional plots revealed that, although many of the surfaces relating $E$ and $P$ to satisfaction could be interpreted from the perspective of P-E fit theory, most were substantially more complex than the highly restricted models depicted by the fit and profile similarity measures. When the constraints imposed by these measures were relaxed, the average proportion of variance explained in job satisfaction was nearly tripled. 


\section{The Present Study}

In this study, we reanalyzed data from the French et al. (1982) study, using the Edwards (in press) procedure to clarify the relationship between $E, P$, and strain. We first reproduce core results reported by French et al., thus providing a baseline for comparing subsequent results. Next, we report confirmatory tests of the restricted models represented by French et al's fit measures to determine whether the conditions required to support these models are met. Finally, we report exploratory analyses, supplemented by cross-validation, to clarify the relationship between $E, P$, and strain. These exploratory analyses were essential, because the vast majority of the restricted models underlying the fit measures were rejected. Cross-validation was also required to identify exploratory models that transcend a single sample and, hence, are likely to be generalizable and theoretically meaningful. Taken together, our analyses resolve ambiguities in the results reported by French et al. and reveal many relationships between $E, P$, and strain that, although consistent with P-E fit theory, are substantially more complex than can be depicted by the fit measures used by French et al.

\section{Method}

The sample, measures, and data collection procedures used in the French et al. (1982) study are described in detail in Caplan et al. (1980). Only a brief description is provided here.

\section{Sample}

French et al. (1982) collected data from respondents in 23 occupations at 67 different sites. Occupations ranged from blue collar (e.g., forklift driver, machine-paced assembler, delivery service courier, and tool-and-die maker), to between blue and white collar (e.g., electronic technician, police officer, train dispatcher, and industrial supervisor), to white collar (e.g., air traffic controller, programmer, accountant, engineer, scientist, professor, administrator, and family physician). These occupations were chosen because they represented a wide range of job characteristics, had exhibited high levels of psychosomatic strain in previous research, and involved minimal exposure to physical and chemical hazards, thereby permitting a focus on social and psychological hazards of work. The sample was exclusively male, because of the difficulty of obtaining adequate samples of female respondents across all occupations at the time the data were collected (1972-1973). The number of respondents in each occupation ranged from 27 to 253 , yielding a total sample size of 2,010 .

To analyze P-E fit relationships, French et al. (1982) used a random stratified subsample of 318 , consisting of about 14 respondents from each occupation that were drawn from the full sample of 2,010 . This subsample was used for two main reasons. First, French et al. hypothesized that P-E fit relationships would differ across occupations. This was supported by follow-up analyses using the full sample, which found significant moderator effects based on occupational membership (French et al., 1982, pp. 83-89). Although these occupational differences are of interest themselves, the primary P-E fit analyses were intended to detect general theoretical relationships that were not specific to a particular occupational group. If the entire sample had been used, results would have been biased in favor of certain occupations, most notably supervisory, administrative, and professional positions. Second, by using the subsample of 318 , enough respondents were retained to form a second, nonoverlapping stratified subsample of equal size for cross-validation. For these reasons, and also to maintain comparability with French et al., we used the initial subsample to repro- duce their results and to conduct confirmatory tests of the models underlying their fit measures. We later incorporated the second subsample for exploratory analyses and cross-validation and then combined the two subsamples to estimate the final exploratory equations.

\section{Data Collection Procedure}

Data were collected using a self-administered questionnaire, which could be completed in about $\mathrm{I}$ hr by a person with a high school education. Most respondents were informed of the study at their places of work, usually by a letter indicating management and union endorsement and ensuring that responses would be anonymous and confidential. Response rates varied by occupation, ranging from $25 \%$ for certain blue-collar occupations to $50 \%-75 \%$ for police officers and university professors to nearly $100 \%$ for train dispatchers.

\section{Measures}

For our study, we analyzed two sets of measures contained in French et al.'s (1982) questionnaire. One set consisted of parallel items measuring environmental supplies $(E)$ and respondent preferences $(P)$ regarding four job dimensions, including job complexity (six item pairs), role ambiguity (four item pairs), responsibility for persons (four item pairs), and quantitative work load (seven item pairs). An example item pair for quantitative work load was "How much work load do you have?" and "How much work load would you like to have?" with responses ranging from very little (1) to a great deal (5). Job complexity items used a 7-point scale, whereas role ambiguity, responsibility for persons, and quantitative work-load items used 5-point response scales. Item responses were averaged to yield $E$ and $P$ scores.

Before our analyses, we scale centered $E$ and $P$ measures by subtracting the midpoints of their scales ( 4 for job complexity and 3 for role ambiguity, responsibility for persons, and quantitative work load). This transformation reduced multicollinearity in subsequent regression analyses and provided a meaningful interpretation of coefficients on $E$ and $P$ when higher order terms were included (i.e., the slopes of $E$ and $P$ at the midpoint of their respective scales; see Aiken \& West, 1991; Cronbach, 1987, Edwards, in press; Jaccard, Turrisi, \& Wan, 1990). Although this transformation was not conducted by French et al. (1982), it did not influence scores on fit measures, their correlations with strain, or tests of the constraints they impose; therefore, this transformation did not compromise the comparability between our results and those reported by French et al.

The second set of measures consisted of seven indexes of psychological strain-including job dissatisfaction (4 items), work-load dissatisfaction ( 3 items), boredom ( 3 items), depression (6 items), anxiety ( 4 items), irritation ( 3 items), and somatic complaints (10 items)-measured on 3- to 5-point response scales. Sample items included "I am unhappy about my current work load," for work-load dissatisfaction; "The work on my job feels dull," for boredom; "I feel sad," for depression; and "You had trouble sleeping at night," for somatic complaints. We averaged items for each index to yield an overall score.

\section{Analysis}

Reproduction of French et al. (1982). We calculated fit, deficiency, excess, poor fit, and fit squared measures for each of the four job dimensions, following the procedures described earlier. We then calculated correlations between these measures and our seven indexes of strain. These analyses reproduced those reported by French et al. (1982) and Caplan et al (1980) and provided a benchmark for comparing subsequent results.

French et al. (1982) also tested the increment in variance explained by the deficiency, excess, and poor fit measures, after controlling for $E$ 
and $P$. We omitted these analyses, because they do not support the restricted models underlying these measures (as depicted in Figure 1) unless the coefficients on $E$ and $P$ happen to be zero. For example, consider a regression equation in which poor fit is significant after controlling for $E$ and $P$. If the coefficients on $E$ and poor fit are positive and equal and the coefficient on $P$ is negative and of the same magnitude, then this equation is algebraically equivalent to the excess measure. Conversely, if the signs of the coefficients on $E$ and $P$ are reversed, then the equation is equivalent to the deficiency measure. Caplan et al. (1980) also tested the increment in variance explained by fit squared, after controlling for fit. In this case, a nonzero coefficient on fit moves the minimum of the $U$-shaped curve represented by fit squared (Figure 1i) away from the point where $E$ and $P$ are equal. By analyzing the bivariate relationships of deficiency, excess, poor fit, and fit squared with strain, we effectively constrained the coefficients on $E$ and $P$ to zero, as implied by the models underlying these measures. We return to this point later, when we discuss the implications of the present study for P-E fit research.

Confirmatory analyses. We used the Edwards (in press) procedure to conduct confirmatory tests of the restricted models represented by the fit, deficiency, excess, poor fit, and fit squared measures. This procedure begins with the researcher deriving an unconstrained regression equation for each fit measure. To do this, an equation containing the fit measure as a single predictor is expanded to show its implied constraints. These constraints are then systematically relaxed, and any missing lower order terms are added. Constraints that we identified for the five fit measures, along with their corresponding constrained and unconstrained equations, are summarized in Table 1.

First, consider the fit measure, which uses the algebraic difference between $E$ and $P$ as a single predictor of strain ( $S$; $e$ represents a random disturbance term):

$$
S=b_{0}+b_{1}(E-P)+e .
$$

Expanding this equation yields

$$
S=b_{0}+b_{1} E-b_{1} P+e .
$$

This shows that the fit measure implicitly constrains the coefficients on $E$ and $P$ to be equal in magnitude but opposite in sign. The corresponding unconstrained equation simply contains $E$ and $P$ as separate predictors:

$$
S=b_{0}+b_{1} E+b_{2} P+e .
$$

Equation 3 relaxes the constraint on the coefficients on $E$ and $P$, allowing them to independently take on whatever values maximize the variance explained in strain.

Next, consider an equation containing the poor fit measure, which represents the absolute difference between $E$ and $P$. Although the constraints imposed by this measure may not be readily apparent, they become obvious by considering the following piecewise linear equation (Neter, Wasserman, \& Kutner, 1989), which is mathematically equivalent to using the absolute difference between $E$ and $P$ to predict strain:

$$
S=b_{0}+b_{1}(1-2 W)(E-P)+e .
$$

$W$ represents a dummy variable that equals 0 when $E>P$, that equals I when $E<P$, and that is randomly set to 0 or 1 when $E=P$. Therefore, when $E-P$ is positive, the term $1-2 W$ reduces to $l$ and the sign of $E-$ $P$ is unaltered, but when $E-P$ is negative, $1-2 W$ reduces to -1 and the sign of $E-P$ is reversed, yielding the same effect as an absolute value transformation. Note that when $E-P=0$, the term $(1-2 W)(E-P)$ becomes 0 , whether $W$ equals 0 or 1 . Expanding and rearranging Equation 4 yields

$$
S=b_{0}+b_{1} E-b_{1} P-2 b_{1} W E+2 b_{1} W P+e .
$$

Now consider an equation containing $E, P, W, W E$, and $W P$ as separate predictors ( $W$ is included because it is a component of the product terms $W E$ and $W P$; see Cohen \& Cohen, 1983):

$$
S=b_{0}+b_{1} E+b_{2} P+b_{3} W+b_{4} W E+b_{5} W P+e .
$$

Comparing Equations 5 and 6 reveals that the poor fit measure imposes four constraints: (a) The coefficients on $E$ and $P$ are equal in magnitude but opposite in sign; (b) the coefficients on $W E$ and $W P$ are equal in magnitude but opposite in sign; (c) the coefficient on $W E$ is twice as large as the coefficient on $E$, but opposite in sign; and (d) the coefficient on $W$ is 0 .

Next, consider the deficiency measure, which equals $E-P$ when $E<P$ and equals 0 when $E>P$. This is represented by the following regression equation:

$$
S=b_{0}-b_{1} W(E-P)+e .
$$

When $E<P, W=1$ and $W(E-P)$ simplifies to $E-P$, but when $E>P$, $W=0$ and $W(E-P)=0$, yielding the same effect as the deficiency measure. As before, when $E=P, E-P=0$ and $W(E-P)$ again equals 0 , regardless of $W$. Expanding Equation 7 yields

$$
S=b_{0}-b_{1} W E+b_{1} W P+e .
$$

Comparing Equation 8 to Equation 6 reveals that the deficiency measure imposes four constraints: (a) The coefficients on $W E$ and $W P$ are equal in magnitude but opposite in sign, (b) the coefficient on $E$ is 0 , (c) the coefficient on $P$ is 0 , and (d) the coefficient on $W$ is 0 .

Now consider the excess measure, which equals $E-P$ when $E \geq P$ and 0 when $E<P$. This is represented by the following regression equation:

$$
S=b_{0}+b_{1}(1-W)(E-P)+e .
$$

In this case, when $E>P, W=0$ and $(1-W)(E-P)$ simplifies to $E-P$, but when $E<P, W=1$ and $(1-W)(E-P)=0$. As before, when $E=P$, $E-P=0$ and $(1-W)(E-P)=0$, independent of $W$ Expanding and rearranging Equation 9 yields

$$
S=b_{0}+b_{1} E-b_{1} P-b_{1} W E+b_{1} W P+e .
$$

Comparing Equation 10 to Equation 6 shows that the excess measure imposes four constraints: (a) The coefficients on $E$ and $P$ are equal in magnitude but opposite in sign, (b) the coefficients on $W E$ and $W P$ are equal in magnitude but opposite in sign, (c) the coefficients on $E$ and $W E$ are equal in magnitude but opposite in sign, and (d) the coefficient on $W$ is 0 .

Finally, the constraints imposed by the fit squared measure can be derived by considering an equation containing the squared difference between $E$ and $P$ as a single predictor:

$$
S=b_{0}+b_{1}(E-P)^{2}+e .
$$

Expanding and rearranging Equation 11 yields

$$
S=b_{0}+b_{1} E^{2}-2 b_{1} E P+b_{1} P^{2}+e .
$$

Now consider an equation containing $E, P, E^{2}, E P$, and $P^{2}$ as separate predictors ( $E$ and $P$ are included because they are components of the curvilinear and interactive terms $E^{2}, E P$, and $P^{2}$; Cohen $\&$ Cohen, 1983):

$$
S=b_{0}+b_{1} E+b_{2} P+b_{3} E^{2}+b_{4} E P+b_{5} P^{2}+e .
$$

Comparing Equation 13 to Equation 12 reveals that the fit squared measure imposes four constraints: (a) The coefficients on $E^{2}$ and $P^{2}$ are equal; (b) the coefficients on $E^{2}, E P$, and $P^{2}$ sum to 0 (given the first constraint, this is equivalent to stating that the coefficient on $E P$ is 
JEFFREY R. EDWARDS AND R. VAN HARRISON

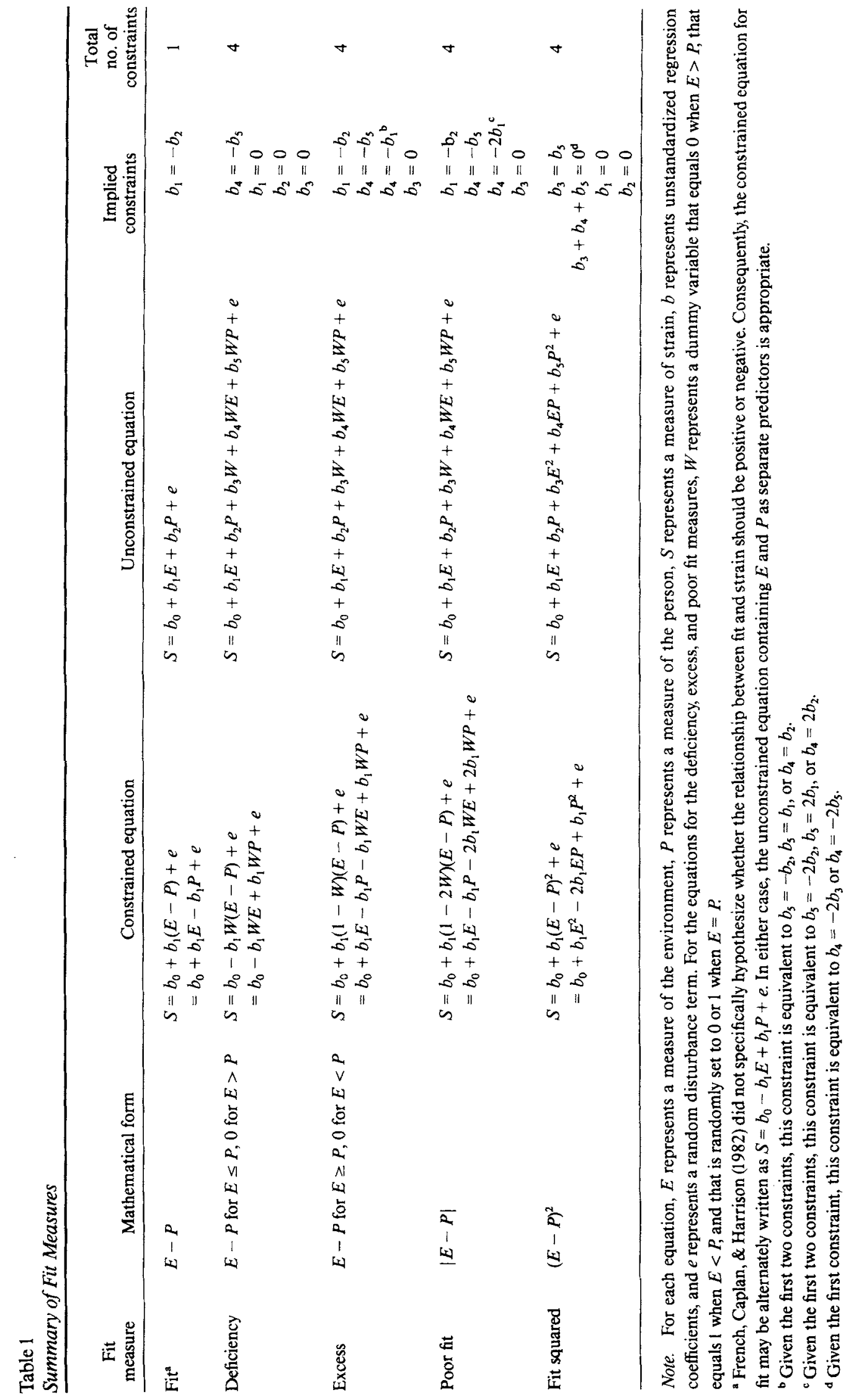


twice as large as the coefficient on either $E^{2}$ or $P^{2}$ but opposite in sign; see Edwards, in press); (c) the coefficient on $E$ is 0 ; and (d) the coefficient on $P$ is 0 .

Once we identified the appropriate unconstrained equations, we estimated them and tested them for significance. We then tested individual coefficients to determine whether they were significant and in the predicted direction. Next, we tested the set of constraints imposed by each measure, using the HYPOTHESIS subroutine of the Multivariate General Linear Hypothesis (MGLH) module of SYSTAT (Wilkinson, 1990b) to determine whether the estimated coefficients conformed to the prescribed pattern (this is equivalent to testing the difference in $R^{2}$ between the constrained and unconstrained equations, although the procedure used here also allowed tests of individual constraints). Finally, we tested sets of terms one order higher than those in the unconstrained equations to determine whether each equation was adequately complex to represent the underlying surface. Support for the model underlying the fit measure was inferred if (a) the overall unconstrained equation was significant, (b) the appropriate coeffcients were significant and in the right direction, (c) the imposed constraints were not rejected, and (d) no significant higher order terms beyond those specified by the unconstrained equation were found (Edwards, in press). It should be emphasized that these analyses tested highly restricted versions of the fit, deficiency, excess, poor fit, and fit squared models, as depicted by their corresponding fit measures (see Figure 1). Failure to support these models did not preclude less restricted versions that were conceptually consistent with P-E fit theory, as our subsequent analyses show.

Support for the constraints imposed by the fit, deficiency, excess, poor fit, and fit squared measures involved establishing that the difference in the squared multiple correlation between the constrained and unconstrained equations was not significant, which required adequate statistical power to detect true differences (Cohen, 1988). With alpha set at .05 and a sample size ranging from 307 to 318 , statistical power was 80 for detecting differences in the squared multiple correlation of about .025 for the fit measure and .0375 for the deficiency, excess, poor fit, and fit squared measures. As we show, actual differences in squared multiple correlations were often larger, averaging .029 for the fit measure and .049 for the deficiency, excess, poor fit, and fit squared measures.

Exploratory analyses. To clarify the joint relationship of $E$ and $P$ with strain, we conducted exploratory analyses supplemented by crossvalidation. First, we used data from the first random subsample to derive 28 exploratory regression equations, 1 for each job dimension predicting each index of strain. We derived these equations by estimating polynomials of progressively higher order (quadratic, cubic, etc.), adding the required variables as a set and stopping when the increment in variance explained was no longer significant. We then repeated this procedure using data from the second random subsample. We compared results for the two subsamples and chose a set of equations common to both subsamples by identifying the highest order polynomial that emerged in both subsamples. For example, if one subsample yielded a quadratic equation and the other successively yielded quadratic, cubic, and quartic equations, we chose the quadratic equation. If no single polynomial was significant in both subsamples, we dropped the equation. For the common equations, we then tested differences between the coefficients for the two subsamples, using the procedure described by Cohen and Cohen (1983, pp. 312-317), to ensure that the equations for the two subsamples were comparable (these tests indicated no significant differences in the coefficients between the two subsamples; all $p s>.05$ ). We reestimated the final equations using data from the combined subsamples.

The procedure just described should be distinguished from crossvalidation as typically practiced in predictive research, in which coeffcients obtained from one sample are used to predict the criterion in another sample. In those situations, the goal is to obtain an estimate of the cross-validated squared multiple correlation, which is represented by the squared correlation between the predicted and actual criterion values in the second sample (Darlington, 1990). In our situation, the goal was to distinguish sampling variability from underlying regularities suggesting meaningful theoretical relationships, using convergent results from multiple samples as evidence for these relationships (Cohen \& Cohen, 1983). This procedure may be more appropriately viewed as replication than as cross-validation, at least as these terms are often used in predictive research (Mosier, 1951).

Controlling Type I error. Because of the substantial number of correlation and regression analyses conducted, some method of controlling Type I error was required. We chose the sequential Bonferroni procedure described by Holm (1979) for its simplicity and demonstrated error control (B. S. Holland \& Copenhaver, 1988; Seaman, Levin, \& Serlin, 1991). This procedure begins with the identification of the family of tests for which Type I error would be controlled. Hochberg and Tamhane (1987) indicated that a family should encompass a set of conceptually related inferences, regardless of their statistical dependence, and Miller (1981) described a family as a group of related observations analyzed within a single mathematical framework. In the present study, the 28 relationships between P-E fit and strain provided a conceptually related set of inferences, and the five fit measures, three unconstrained regression equations, and final exploratory equations represented different mathematical approaches, or frameworks, intended to depict these relationships. Hence, we defined a family as the 28 relationships examined within each mathematical approach, yielding nine families in all.

Next, we listed in ascending order the probability levels for the 28 bivariate or multiple correlations yielded by each approach. We then multiplied the first (i.e., smallest) probability by 28 , representing the total number of tests. If this probability remained below .05 , we then multiplied the next probability by the total number of remaining tests (i.e., 27). This procedure continued sequentially until we had corrected all 28 probabilities, and only those that remained below .05 were considered significant. For each multiple correlation that reached significance, we tested the corresponding regression coefficients using the nominal alpha level (i.e., .05). This procedure held Type I error for each family below .05 , avoided the loss of power associated with the standard Bonferroni correction (B. S. Holland \& Copenhaver, 1988), and permitted comparisons of the relative power of the various fit measures and regression equations.

\section{Results}

\section{Reliability and Intercorrelations of Measures}

Table 2 shows descriptive statistics, reliability estimates (Cronbach's alpha), and intercorrelations for the $E, P$, and strain measures. With the exception of quantitative work load/P, all measures yielded reliabilities of 70 or higher. Corresponding $E$ and $P$ measures were all positively related, with correlations ranging from .135 for quantitative work load to .683 for job complexity. $E$ measures for job complexity, responsibility for persons, and quantitative work load were positively related, as were their respective $P$ measures. All strain measures were also positively related. Within rounding error, these correlations matched those reported by French et al. (1982).

\section{Reproduction of French et al. (1982)}

Table 3 shows correlations between strain and the fit, deficiency, excess, poor fit, and fit squared measures. For job com- 
plexity, poor fit and fit squared demonstrated the strongest relationships with strain, suggesting that strain was lowest at the point of perfect fit and increased symmetrically in either direction. However, correlations for deficiency and excess were opposite in sign but often different in absolute magnitude, implying that the relationship between fit and strain was asymmetric. Furthermore, fit was negatively correlated with boredom, suggesting a monotonic relationship. Similar results were found for quantitative work load and, to a lesser extent, for role ambiguity and responsibility for persons. It should be noted that, in many instances, the correlations reported in Table 3 were smaller than those for the separate $E$ and $P$ measures (Table 2), indicating that the predictive power of the $E$ and $P$ measures taken separately was often diminished by collapsing them into a fit measure.

\section{Confirmatory Analyses}

Results for the unconstrained regression equations for the fit, deficiency, excess, poor fit, and fit squared measures are presented in Table 4 , and $F$ tests of constraints and higher order terms are presented in Table 5. For simplicity, Table 4 shows only those regression equations that reached significance using the sequential Bonferroni procedure, and Table 5 shows tests of constraints and higher order terms only for those equations that were significant and yielded the expected pattern of coefficients (complete tables are available from Jeffrey R. Edwards).

Examining the unconstrained equations for fit shows that the coefficients on $E$ and $P$ were significant and opposite in sign only for quantitative work load predicting work-load dissatisfaction $\left(b_{1}=0.657, b_{2}=-0.808\right)$ and irritation $\left(b_{1}=0.255, b_{2}=\right.$ -0.210 ). Tests of the constraint imposed by the fit measure indicated that the coefficients on $E$ and $P$ did not differ in absolute magnitude in either equation, $F(1,309)=1.42$ and $F(1$, $310)=0.04, p s>.05$. However, significant higher order terms were found for work-load dissatisfaction, $F(3,306)=5.35, p<$ .01 , indicating that the data deviated from the planar surface implied by the fit measure (Figure 1b). In contrast, no significant higher order terms were found for irritation, $F(3,307)=$ $0.91, p>.05$, indicating that the underlying surface, in fact, resembled the plane depicted in Figure 1b, with the caveat that the slope for $E$ was positive and the slope for $P$ was negative. Hence, of the five significant correlations between fit and strain (Table 3), only one supported the model underlying the fit measure.

Results for the unconstrained equation for deficiency, excess, and poor fit indicated that, in 14 of 28 analyses, the overall equation was significant. However, none of these equations yielded the pattern of coefficients corresponding to the deficiency measure (i.e., negative coefficient on $W E$, positive coefficient on $W P$, and nonsignificant coefficients on $E, P$, and $W$ ). In contrast, the pattern of coefficients corresponding to excess and poor fit (i.e., positive coefficients on $E$ and $W P$, negative coefficients on $P$ and $W E$, and nonsignificant coefficient on $W$ ) emerged for job complexity predicting work-load dissatisfaction and anxiety and for responsibility for persons predicting work-load dissatisfaction. Tests of the constraints imposed by the excess measure were rejected for job complexity predicting work-load dissatisfaction, $F(4,302)=2.72, p<.05$, and anxiety, 
Table 3

Correlations Between Fit Measures and Strain

\begin{tabular}{|c|c|c|c|c|c|}
\hline \multirow[b]{2}{*}{$\begin{array}{l}\text { Job dimension \& } \\
\text { strain measure }\end{array}$} & \multicolumn{5}{|c|}{ Fit measure } \\
\hline & Fit & Deficiency & Excess & $\begin{array}{l}\text { Poor } \\
\text { fit }\end{array}$ & $\begin{array}{c}\text { Fit } \\
\text { squared }\end{array}$ \\
\hline \multicolumn{6}{|l|}{ Job complexity } \\
\hline Job dissatisfaction & -.035 & $-.194^{*}$ & $.185^{*}$ & $.311^{* *}$ & $.228^{* *}$ \\
\hline Work-load dissatisfaction & .154 & -.034 & $.325^{* *}$ & $.264^{* *}$ & $.190^{*}$ \\
\hline Boredom & $-.256^{* *}$ & $-.379^{* *}$ & .017 & $.360^{* *}$ & $.322^{* *}$ \\
\hline Depression & .032 & -.088 & $.171^{*}$ & $.204^{* *}$ & .146 \\
\hline Anxiety & .063 & -.076 & $.213^{* *}$ & $.223^{* *}$ & $.171^{*}$ \\
\hline Irritation & .095 & -.017 & $.194^{*}$ & .155 & .068 \\
\hline Somatic complaints & -.113 & $-.190^{*}$ & .038 & $.202^{* *}$ & $.174^{*}$ \\
\hline \multicolumn{6}{|l|}{ Role ambiguity } \\
\hline Job dissatisfaction & .075 & -.014 & $.169^{*}$ & .137 & .087 \\
\hline Work-load dissatisfaction & .037 & -.022 & .104 & .097 & .080 \\
\hline Boredom & .042 & -.035 & .132 & .130 & .128 \\
\hline Depression & .058 & .005 & .107 & .072 & .050 \\
\hline Anxiety & .092 & .071 & .083 & -.011 & -.046 \\
\hline Irritation & .123 & .074 & .142 & .029 & .022 \\
\hline Somatic complaints & .032 & .030 & .021 & -.015 & -.027 \\
\hline \multicolumn{6}{|l|}{ Responsibility for persons } \\
\hline Job dissatisfaction & -.135 & $-.184^{*}$ & .022 & $.212^{* *}$ & $.204^{* *}$ \\
\hline Work-load dissatisfaction & .072 & .005 & $.176^{*}$ & .088 & .096 \\
\hline Boredom & $-.236^{* *}$ & $-.287^{* *}$ & -.031 & $.295^{* *}$ & $.303^{* *}$ \\
\hline Depression & .009 & -.023 & .070 & .063 & .062 \\
\hline Anxiety & .052 & .007 & .121 & .058 & .041 \\
\hline Irritation & .055 & .040 & .061 & -.011 & .006 \\
\hline Somatic complaints & -.100 & -.098 & -.061 & .074 & .051 \\
\hline \multicolumn{6}{|l|}{ Quantitative work load } \\
\hline Job dissatisfaction & .191 & .076 & $.208^{* *}$ & $.188^{*}$ & $.188^{*}$ \\
\hline Work-load dissatisfaction & $.495^{* *}$ & $.260^{* *}$ & $.510^{* *}$ & $.419^{* *}$ & $.404^{* *}$ \\
\hline Boredom & .049 & .019 & .054 & .049 & .043 \\
\hline Depression & $.248^{* *}$ & .160 & $.241^{* *}$ & $.177^{*}$ & $.187^{*}$ \\
\hline Anxiety & .120 & .058 & .126 & .107 & .071 \\
\hline Irritation & $.266^{* *}$ & .145 & $.271^{* *}$ & $.220^{* *}$ & $.199^{* * *}$ \\
\hline Somatic complaints & .047 & .006 & .058 & .060 & .018 \\
\hline
\end{tabular}

Note. Ns ranged from 308 to 313 . Table entries are product-moment correlations. Probability levels for the 28 correlations tested for each fit measure were corrected using the sequential Bonferroni procedure described by Holm (1979).

$* p<.05$. ${ }^{* *} p<.01$.

$F(4,303)=2.53, p<.05$. However, the excess constraints were not rejected for responsibility for persons predicting work-load dissatisfaction, $F(4,305)=2.10, p>.05$. Higher order terms were also not significant, $F(6,299)=1.04, p>.05$, thereby indicating that the surface relating responsibility for persons $/ E$ and responsibility for persons/ $P$ to work-load dissatisfaction resembled Figure If. Constraints for the poor fit measure were rejected for job complexity predicting work-load dissatisfaction, $F(4,302)=5.85, p<.01$, and for responsibility for persons predicting work-load dissatisfaction, $F(4,305)=3.97, p<.01$. However, the poor fit constraints were not rejected for job complexity predicting anxiety, $F(4,303)=2.18, p>.05$, and higher order terms were not significant, $F(6,297)=1.74, p>.05$, indicating that the surface relating job complexity/ $E$ and job complexity/ $P$ to anxiety corresponded to Figure $1 h$. Hence, although the deficiency, excess, and poor fit measures collectively yielded 29 significant correlations with strain, the deficiency model was not supported, and the excess and poor fit models were each supported only once.

Results for the unconstrained equation for fit squared indi- cated that in 13 of 28 analyses, the overall equation was significant. However, the expected pattern of coefficients (i.e., nonsignificant coefficients on $E$ and $P$, positive coefficients on $E^{2}$ and $P^{2}$, and negative coefficient on $E P$ ) was obtained only for responsibility for persons predicting boredom. However, the constraints imposed by the fit squared measure were rejected, $F(4$, $305)=3.34, p<.01$. Hence, although the fit squared measure yielded il significant correlations with strain, the restricted model underlying this measure received no support.

The preceding results strongly indicate that, with few exceptions, the restricted models underlying the fit measures used by French et al. (1982) do not adequately represent the relationship between $E, P$, and strain. This may seem counterintuitive, given that these measures were often significantly correlated with strain. To clarify why these models were not supported, we present in Figures 2 and 3 three-dimensional plots of raw data, surfaces predicted by the five fit measures, and surfaces predicted by the three unconstrained equations, using job complexity predicting boredom and quantitative work load predicting work-load dissatisfaction as examples. These examples were 


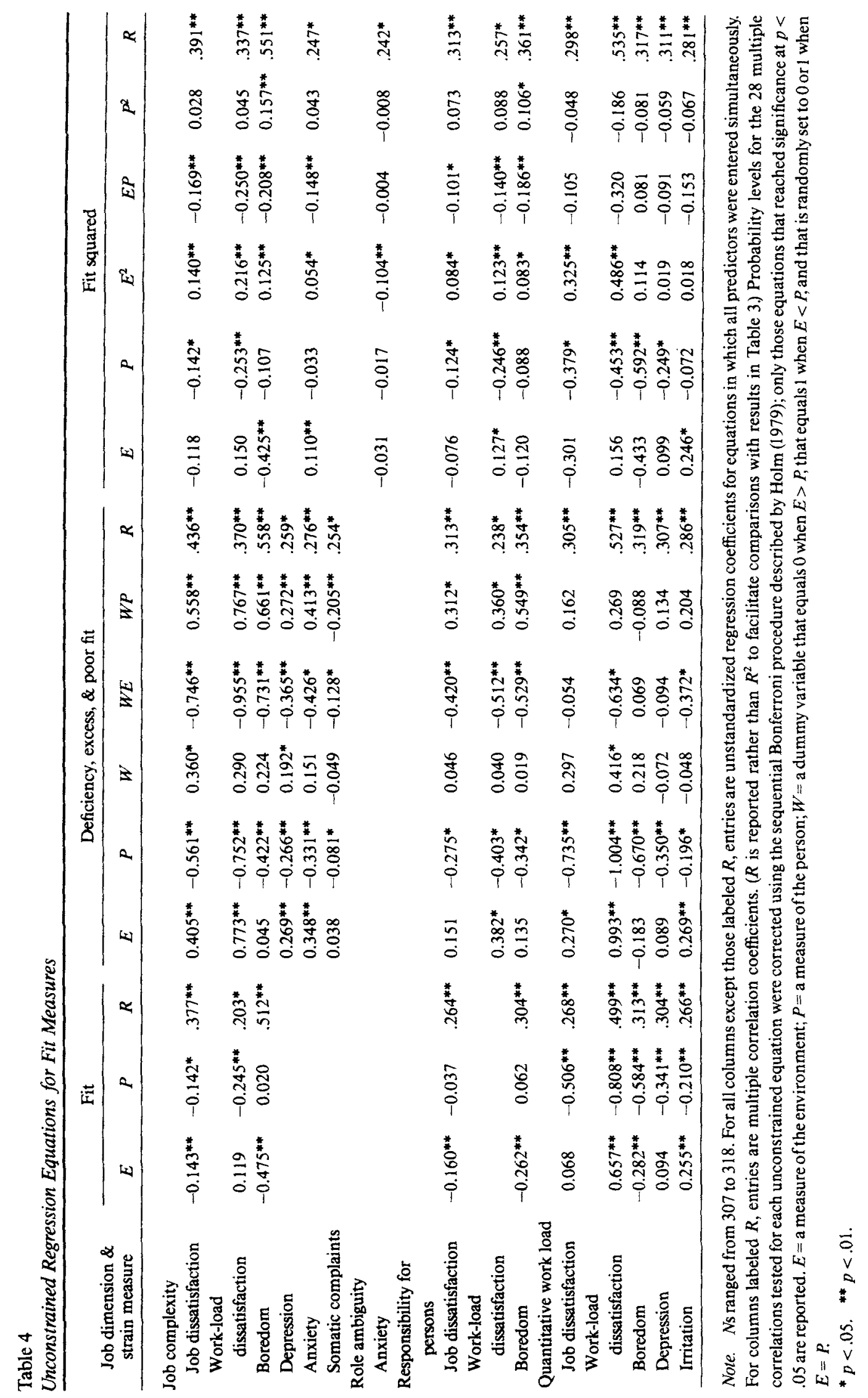


Table 5

Tests of Constraints and Higher Order Terms

\begin{tabular}{|c|c|c|c|c|c|c|c|}
\hline \multirow[b]{2}{*}{ Strain measure } & \multicolumn{2}{|c|}{ Fit $^{\mathbf{a}}$} & \multicolumn{3}{|c|}{ Excess \& poor fit ${ }^{b}$} & \multicolumn{2}{|c|}{ Fit squared ${ }^{c}$} \\
\hline & $\begin{array}{c}\text { Fit } \\
\text { constraint }\end{array}$ & $\begin{array}{l}\text { Higher } \\
\text { order } \\
\text { terms }\end{array}$ & $\begin{array}{c}\text { Excess } \\
\text { constraints }\end{array}$ & $\begin{array}{c}\text { Poor fit } \\
\text { constraints }\end{array}$ & $\begin{array}{l}\text { Higher } \\
\text { order } \\
\text { terms }\end{array}$ & $\begin{array}{l}\text { Fit squared } \\
\text { constraints }\end{array}$ & $\begin{array}{l}\text { Higher } \\
\text { order } \\
\text { terms }\end{array}$ \\
\hline \multicolumn{8}{|l|}{ Job complexity } \\
\hline Work-load dissatisfaction & & & $2.72^{*}$ & $5.85^{* *}$ & 0.85 & & \\
\hline Anxiety & & & $2.53^{*}$ & 2.18 & 1.74 & & \\
\hline \multicolumn{8}{|l|}{ Responsibility for persons } \\
\hline Work-load dissatisfaction & & & 2.10 & $3.97^{* *}$ & 1.04 & & \\
\hline Boredom & & & & & & $3.34^{* *}$ & 1.20 \\
\hline \multicolumn{8}{|l|}{ Quantitative work load } \\
\hline Work-load dissatisfaction & 1.42 & $5.35^{* *}$ & & & & & \\
\hline Irritation & 0.04 & 0.91 & & & & & \\
\hline
\end{tabular}

Note. $\quad N$ s ranged from 308 to 313 . Table entries are $F$ ratios. For each model, tests are only reported for those equations that yielded significant multiple correlations and the predicted pattern of coefficients. $E=$ measure of environment; $P=$ measure of person; $W=$ a dummy variable that equals 0 when $E>P$, that equals 1 when $E<P$, and that is randomly set to 0 or 1 when $E=P$.

${ }^{a}$ For fit, a single constraint was tested, that is, the coefficients on $E$ and $P$ are equal in magnitude but opposite in sign $\left(b_{1}=-b_{2}\right)$. Higher order terms were tested as a set and included $E^{2}, E P$, and $P^{2}$, representing a quadratic model.

${ }^{b}$ For excess, four constraints were tested as a set: (a) The coefficients on $E$ and $P$ are equal in magnitude but opposite in sign $\left(b_{1}=-b_{2}\right)$, (b) the coefficients on $W E$ and $W P$ are equal in magnitude but opposite in sign $\left(b_{4}=-b_{5}\right)$, (c) the coefficients on $E$ and $W E$ are equal in magnitude but opposite in $\operatorname{sign}\left(b_{4}=-b_{1}\right)$, and (d) the coefficient on $W\left(b_{3}\right)$ is 0 . For poor fit, four constraints were tested as a set: (a) The coefficients on $E$ and $P$ are equal in magnitude but opposite in sign $\left(b_{1}=-b_{2}\right) ;($ b) the coefficients on $W E$ and $W P$ are equal in magnitude but opposite in sign $\left(b_{4}=-b_{5}\right)$; (c) the coefficient on $W E$ is twice as large as the coefficient on $E$, but opposite in sign $\left(b_{4}=-2 b_{1}\right)$; and (d) the coefficient on $W\left(b_{3}\right)$ is 0 . For both excess and poor fit, higher order terms were tested as a set and included $E^{2}, E P, P^{2}$, and the product of $W$ with these terms $\left(W E^{2}, W E P\right.$, and $\left.W P^{\mathrm{Z}}\right)$, which allows independent curvature and tilt in the triangular surfaces on either side of the $E=P$ line.

${ }^{c}$ For fit squared, four constraints were tested as a set: (a) The coefficients on $E^{2}$ and $P^{2}$ are equal $\left(b_{3}=b_{5}\right)$; (b) the coefficients on $E^{2}, E P$, and $P^{2}$ sum to zero $\left(b_{3}+b_{4}+b_{5}=0\right)$; (c) the coefficient on $E\left(b_{1}\right)$ is 0 ; and (d) the coefficient on $P\left(b_{2}\right)$ is 0 . Higher order terms were tested as a set and included $E^{3}, E^{2} P, E P^{2}$, and $P^{3}$, representing a cubic model.

${ }^{*} p<.05 .{ }^{* *} p<.01$.

chosen because they yielded the strongest relationships across the five fit measures but, nonetheless, rejected the models underlying these measures in every case. For both examples, raw data were plotted using distance-weighted least squares, which produces a locally weighted surface that flexes to fit the data (McLain, 1974; Wilkinson, 1990a).

Raw data for job complexity predicting boredom (Figure 2a) indicated that boredom not only decreased as either $E$ or $P$ increased but also was lower in the region where $E$ and $P$ were approximately equal. This surface differed substantially from those predicted by the fit and deficiency measures (Figures $2 b$ and $2 \mathrm{c}$ ), even though both yielded significant correlations with boredom. Surfaces predicted by the poor fit and fit squared measures (Figures $2 \mathrm{e}$ and $2 \mathrm{f}$ ) corresponded somewhat more closely to the raw data, but both indicated constant levels of boredom along the $E=P$ line, which is clearly not the case. Of the unconstrained equations, the fit squared equation seemed to best represent the data (Figure $2 \mathrm{i}$ ).

Raw data for quantitative work load predicting work-load dissatisfaction (Figure 3a) indicated that dissatisfaction was lowest in the region where $E$ and $P$ were approximately equal and increased in either direction, with a more pronounced increase where $E$ exceeded $P$ (i.e., where actual work load exceeded pre- ferred work load). This surface was notably different from those predicted by the fit, deficiency, and excess measures (Figures $3 b, 3 c$, and $3 d$, respectively), even though each yielded signifcant correlations with work-load dissatisfaction. The surfaces predicted by the poor fit and fit squared measures (Figures $3 \mathrm{e}$ and 3f) were somewhat better, but both inappropriately forced symmetry on either side of the $E=P$ line. Of the unconstrained equations, the fit squared equation again seemed best, although it did not adequately depict the curvature along the $E$ axis. Further inspection revealed that this curvature was caused by a few isolated cases and, hence, should not be given undue consideration in interpreting the overall shape of the surface.

\section{Exploratory Analyses}

Results for the final exploratory equations of the relationship between $E, P$, and strain are reported in Table 6 . Seven equations indicated that strain was linearly related to either $E$ or $P$. In four of these equations, $E$ was positively related to strain (role ambiguity predicting job dissatisfaction, work-load dissatisfaction, depression, and anxiety), whereas in two equations $E$ was negatively related to strain (responsibility for persons predict- 
a. Raw Data

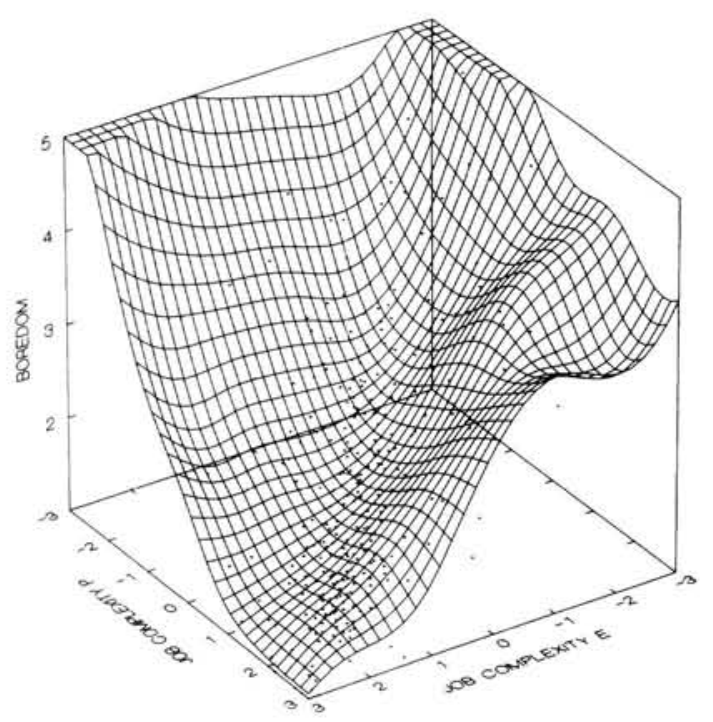

c. Deficiency

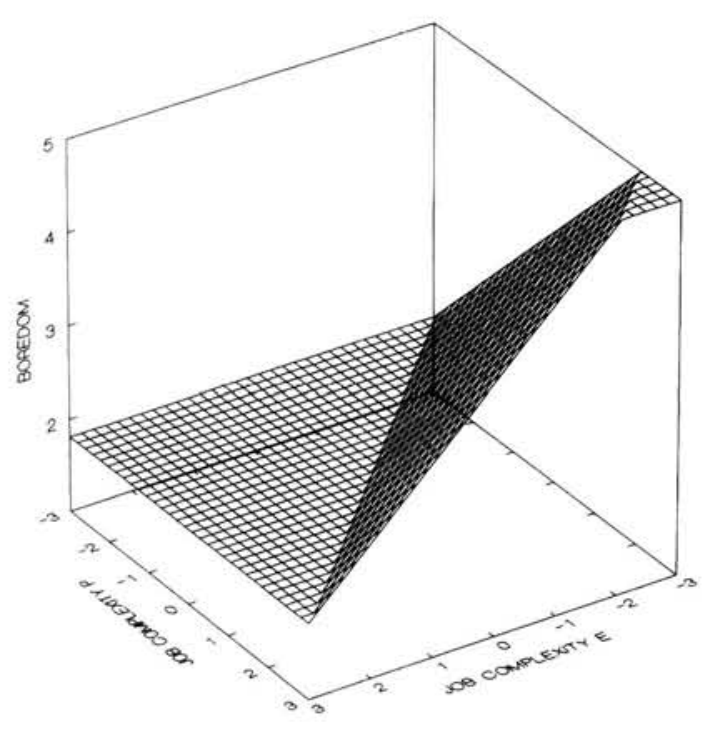

e. Poor Fit

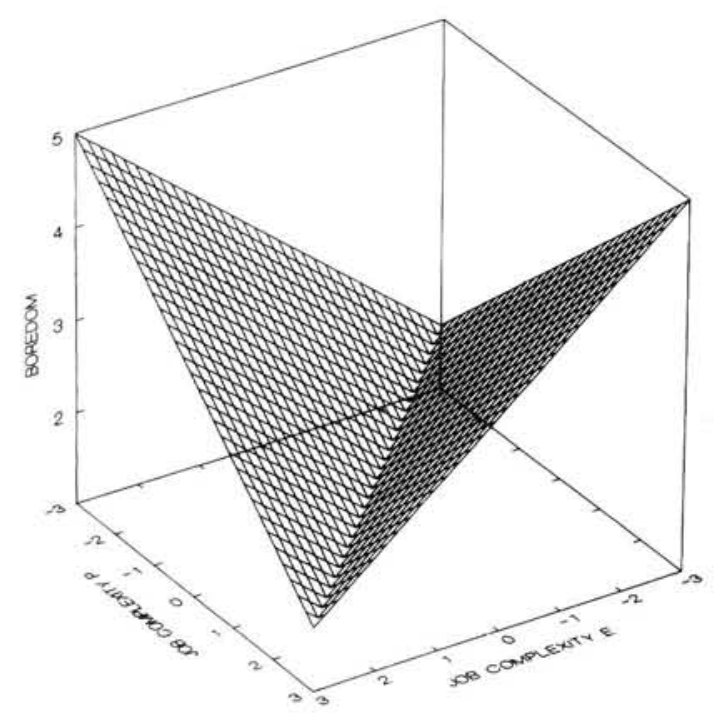

b. Fit

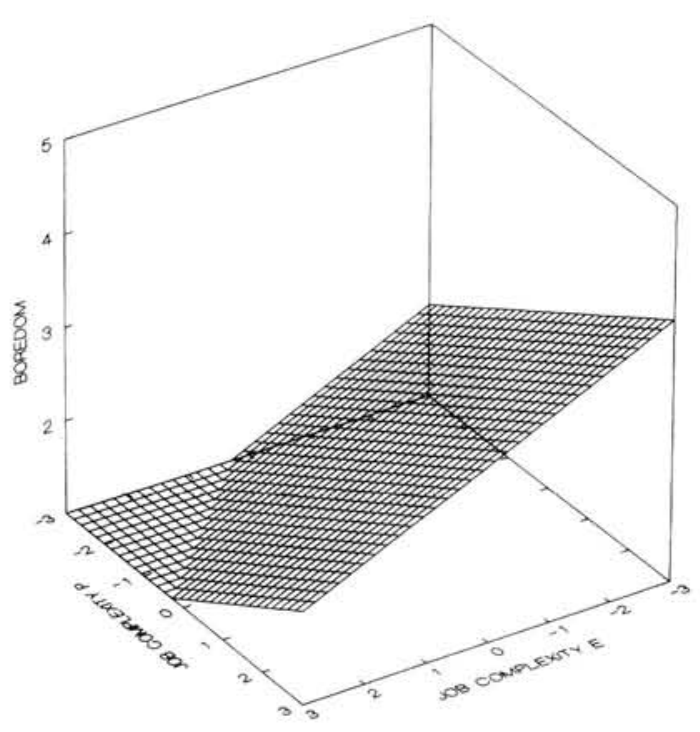

d. Excess

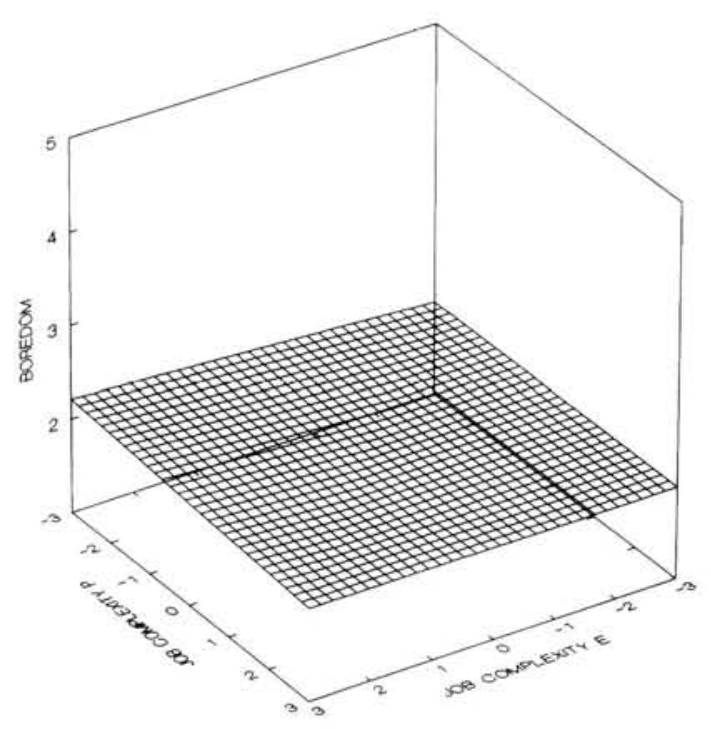

f. Fit Squared

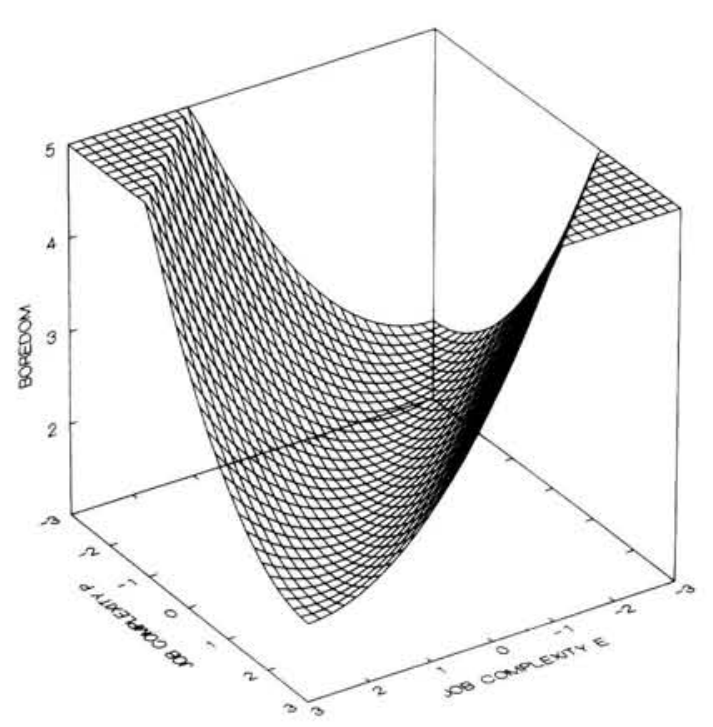


g. Unconstrained Equation for Fit

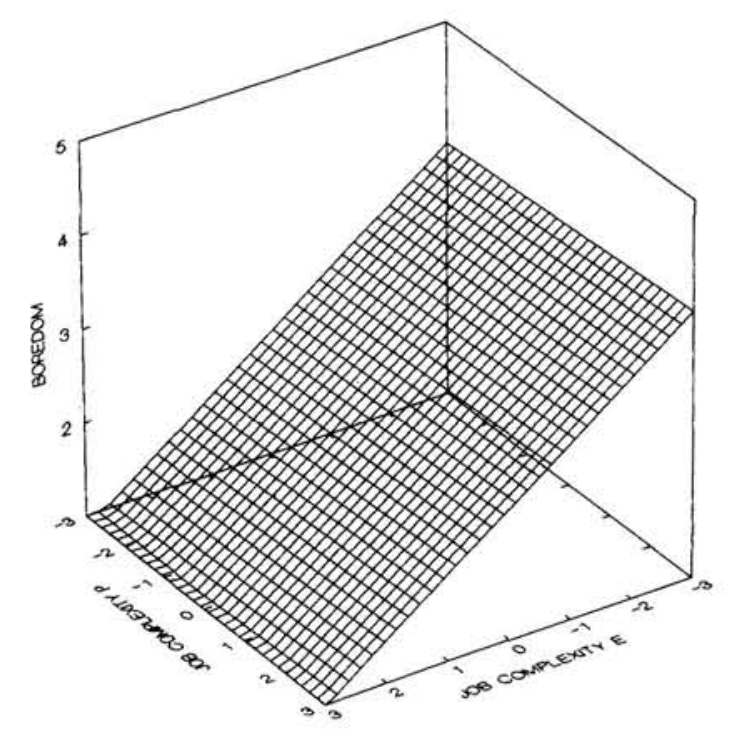

h. Unconstrained Equation for Deficiency. Excess. \& Poor Fit

i. Unconstrained Equation for Fit Squared
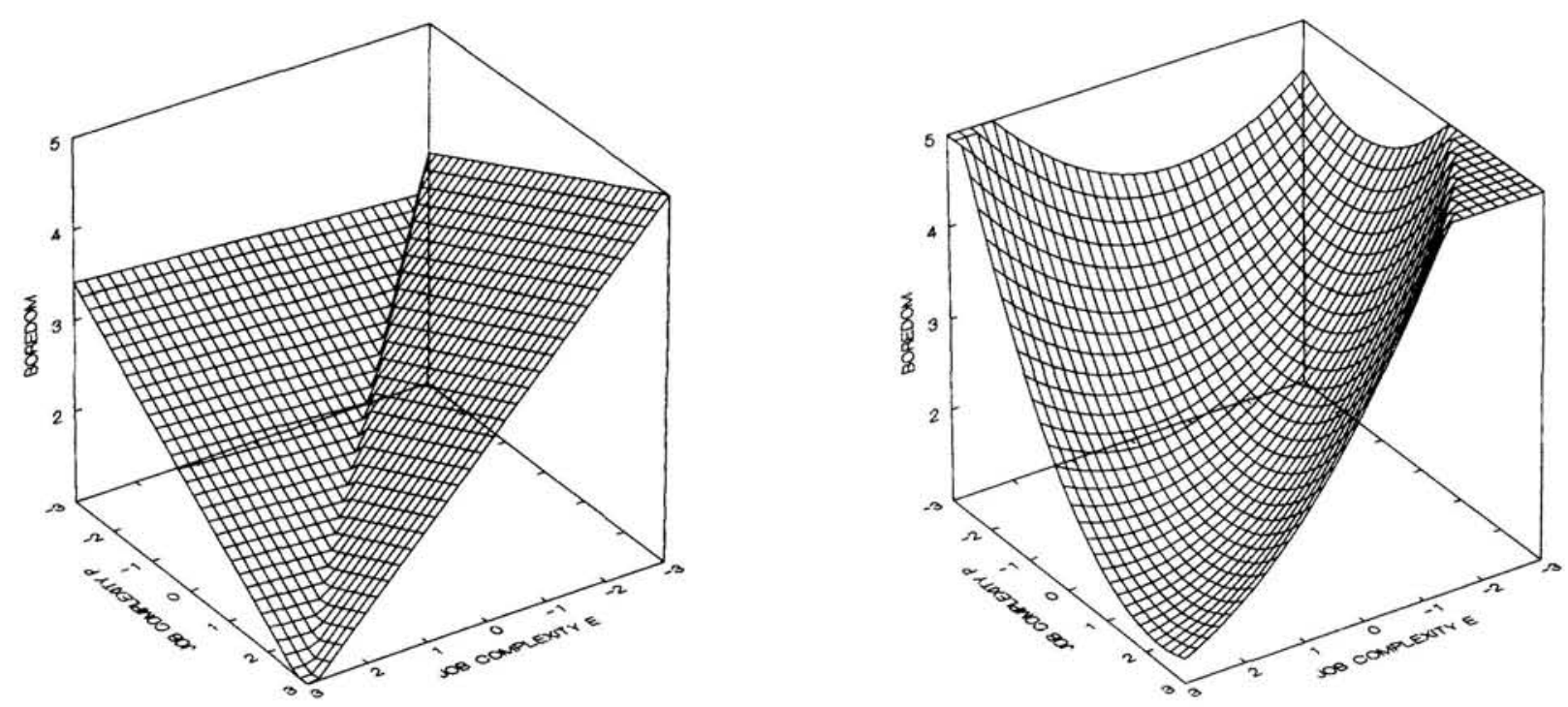

Figure 2 (left and above). Three-dimensional plots of actual and predicted surfaces relating environment $(E)$ and person $(P)$ measures of job complexity to boredom. (Scales for $E$ and $P$ are reversed to allow better viewing of the surfaces.)

ing job dissatisfaction and boredom). One equation indicated a negative relationship between $P$ and strain (quantitative work load predicting job dissatisfaction). Because none of these equations indicated significant relationships for both $E$ and $P$, they provided no evidence for fit.

Three equations yielded significant coefficients for both $E$ and $P$. One equation (quantitative work load predicting boredom) yielded negative coefficients on both $E$ and $P$, indicating that boredom was lowest when quantitative work load $/ E$ and quantitative work load $/ P$ were both high. Although plausible, these relationships were not predicted by P-E fit theory. In contrast, two equations (quantitative work load predicting depres- sion and irritation) yielded a positive coefficient on $E$ and a negative coefficient on $P$, analogous to the fit model. Further analyses indicated that, for quantitative work load predicting irritation, the coefficients on $E$ and $P$ did not significantly differ in absolute magnitude, $F(1,623)=2.55, p>.05$. Hence, the equation for quantitative work load predicting depression supported a weak version of the fit model, in which $E$ and $P$ exhibited opposite but unequal relationships with strain, whereas the equation for quantitative work load predicting irritation supported a strong version of the fit model, in which $E$ and $P$ exhibited opposite but essentially equal relationships with strain. 
a. Raw Data

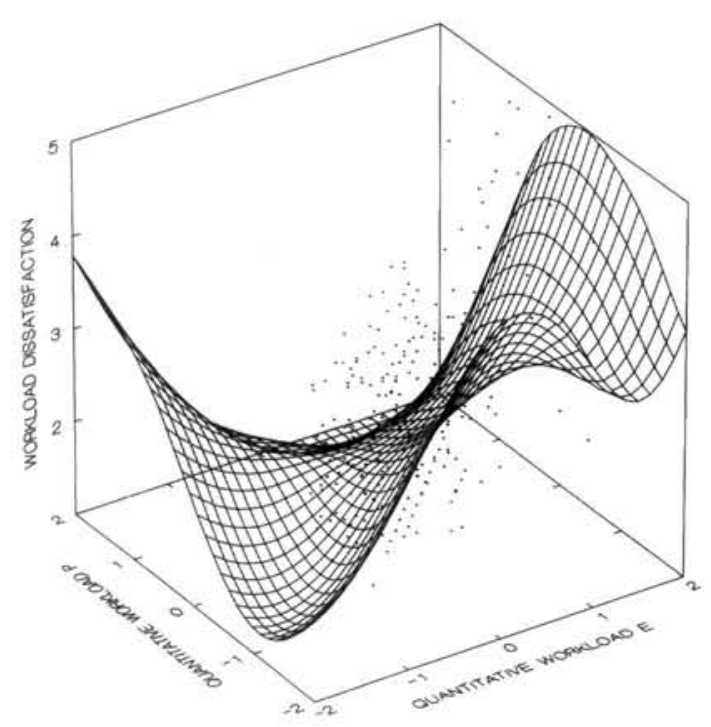

c. Deficiency

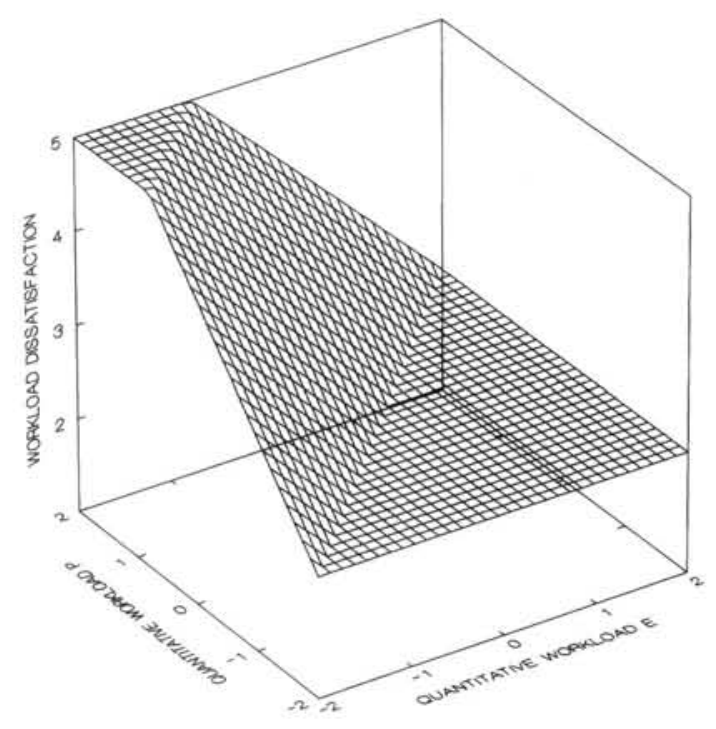

e. Poor Fit

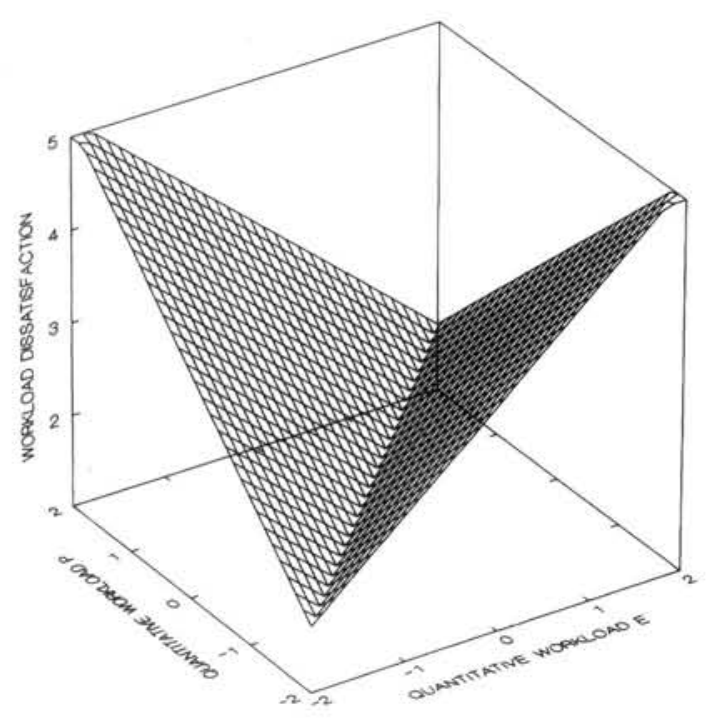

b. Fit

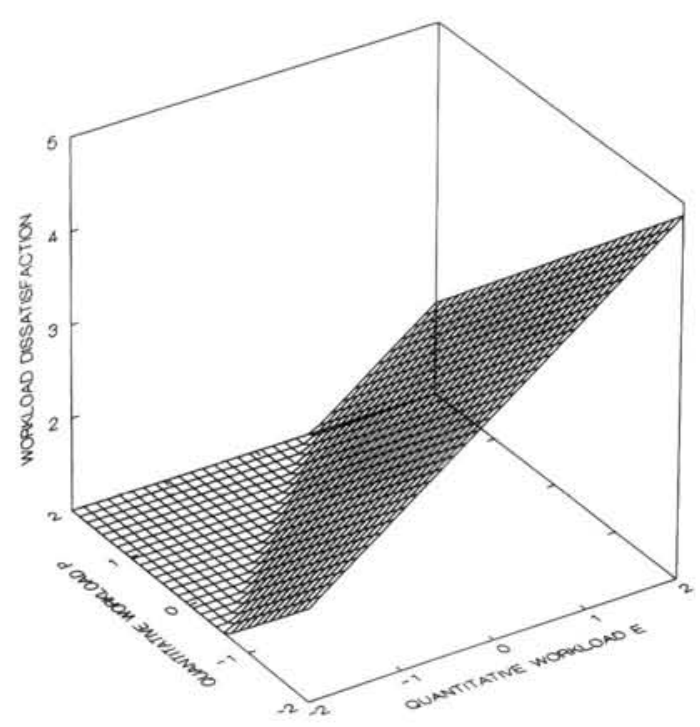

d. Excess

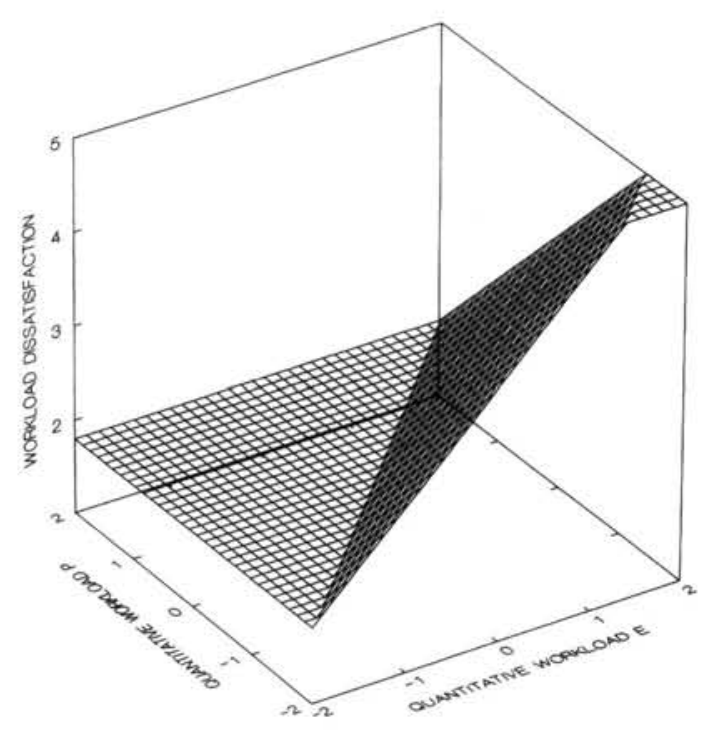

f. Fit Squared

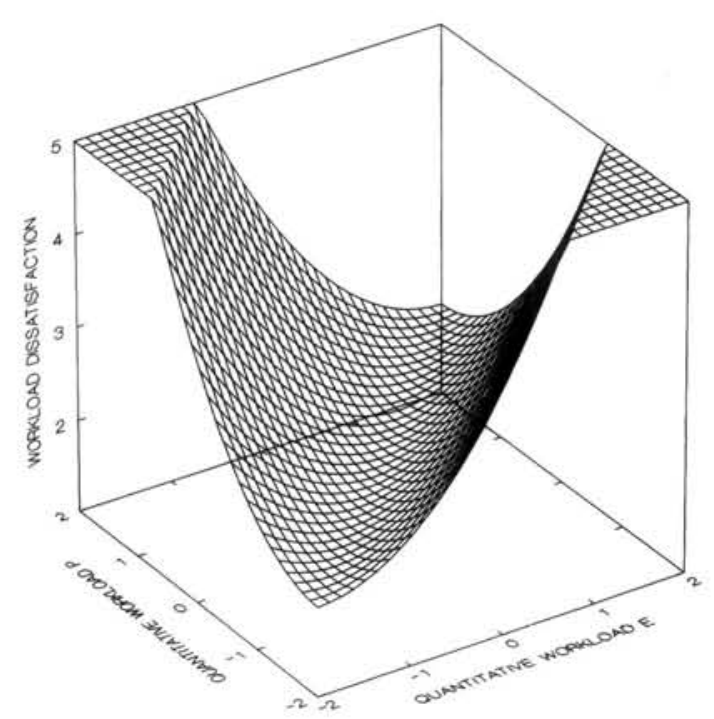




\section{g. Unconstrained Equation for Fit}

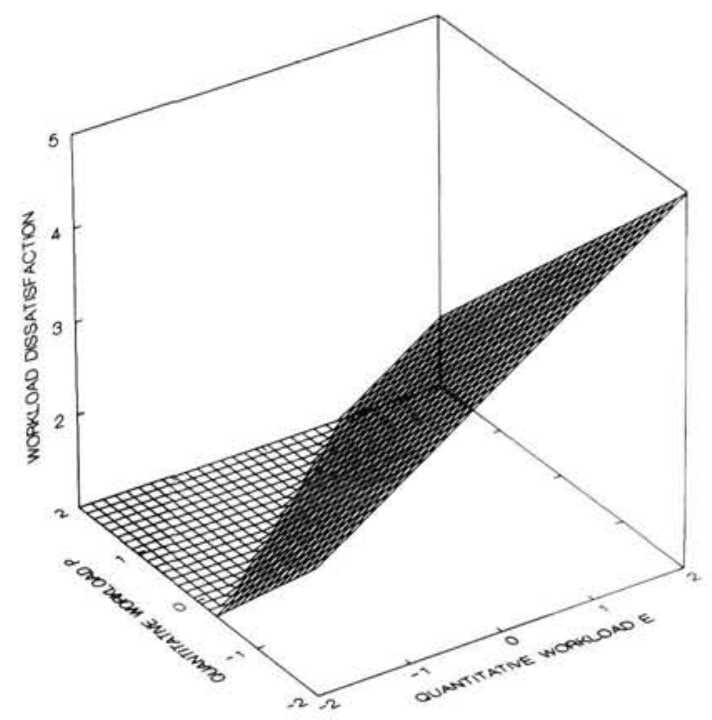

h. Unconstrained Equation for Deficiency. Excess. \& Poor Fit
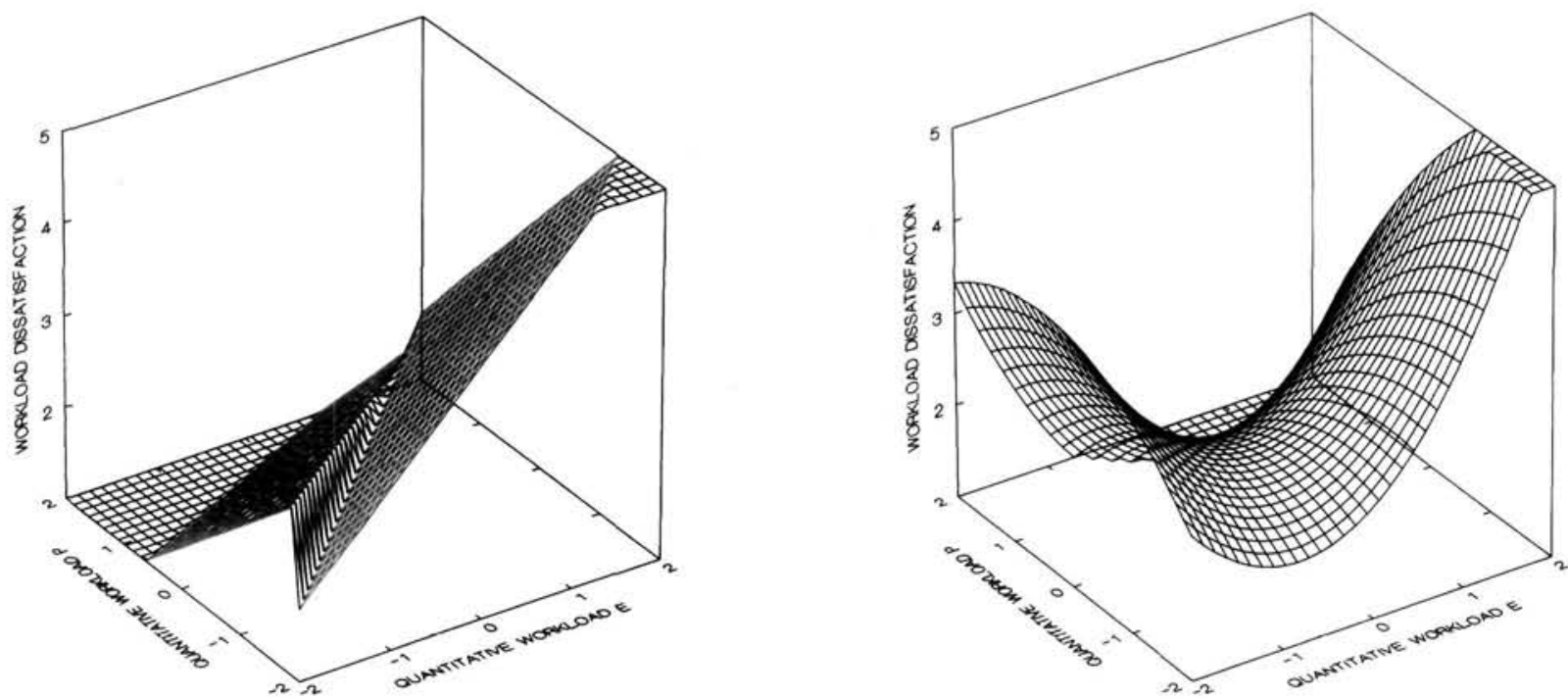

Figure 3 (left and above). Three-dimensional plots of actual and predicted surfaces relating environment $(E)$ and person $(P)$ measures of quantitative work load to work-load dissatisfaction.

Six equations yielded positive coefficients on $E^{2}$, negative coefficients on $E P$, and a mixture of positive, negative, and nonsignificant coefficients on $E, P$, and $P^{2}$. A complete interpretation of these equations and their corresponding surfaces requires the application of response-surface methodology (Box \& Draper, 1987; Khuri \& Cornell, 1987), which is beyond the scope of this article. Nonetheless, salient features of these surfaces can be identified by comparing them with the surface corresponding to the fit squared measure (Figure 1j). For simplicity, we focus on three features. First, consider the slope along the $E=P$ line. This can be represented in equation form by substituting $E$ for $P$ in Equation 13:

$$
\begin{aligned}
S & =b_{0}+b_{1} E+b_{2} E+b_{3} E^{2}+b_{4} E^{2}+b_{5} E^{2}+e \\
& =b_{0}+\left(b_{1}+b_{2}\right) E+\left(b_{3}+b_{4}+b_{5}\right) E^{2}+e \\
& =b_{0}+a_{1} E+a_{2} E^{2}+e .
\end{aligned}
$$

For convenience, we let $a_{1}=b_{1}+b_{2}$ and $a_{2}=b_{3}+b_{4}+b_{5}$. As Equation 14 shows, the slope along the $E=P$ line has a linear component $\left(a_{1} E\right)$ and a curvilinear component $\left(a_{2} E^{2}\right)$. If the fit squared constraints hold, then both $b_{1}$ and $b_{2}$ equal 0 and $b_{3}, b_{4}$, and $b_{5}$ sum to 0 . Consequently, $a_{1}$ and $a_{2}$ are both 0 , indicating that the surface is flat along the $E=P$ line, as in Figure lj. If $a_{1}$ differs from 0 but $a_{2}$ does not, then the surface has a linear slope 
Table 6

Final Exploratory Equations for E, , and Strain

\begin{tabular}{|c|c|c|c|c|c|c|c|}
\hline Strain measure & $E$ & $P$ & $E^{2}$ & $E P$ & $P^{2}$ & $R^{2}$ & $R_{\mathrm{adj}}^{2}$ \\
\hline \multicolumn{8}{|l|}{ Job complexity } \\
\hline Job dissatisfaction & -0.082 & $-0.117^{*}$ & $0.131^{* *}$ & $-0.216^{* *}$ & 0.068 & $.140^{* *}$ & .133 \\
\hline Work-load dissatisfaction & 0.103 & $-0.226^{* *}$ & $0.212^{* *}$ & $-0.207^{* *}$ & 0.023 & $.100^{* *}$ & .093 \\
\hline Boredom & $-0.467^{* *}$ & -0.023 & $0.154^{* *}$ & $-0.206^{* *}$ & $0.124^{* *}$ & $.301^{* *}$ & .296 \\
\hline Depression & 0.023 & -0.029 & $0.060^{* *}$ & $-0.099^{* *}$ & 0.014 & $.044^{* *}$ & .037 \\
\hline Anxiety & $0.096^{* *}$ & -0.009 & $0.047^{* *}$ & $-0.153^{* *}$ & 0.044 & $.058^{* *}$ & .051 \\
\hline \multicolumn{8}{|l|}{ Role ambiguity } \\
\hline Job dissatisfaction & $0.215^{* *}$ & -0.004 & & & & $.044^{* *}$ & .041 \\
\hline Work-load dissatisfaction & $0.252^{* *}$ & -0.006 & & & & $.044^{* *}$ & .041 \\
\hline Depression & $0.138^{* *}$ & 0.011 & & & & $.050^{* *}$ & .047 \\
\hline Anxiety & $0.138^{* *}$ & -0.029 & & & & $.043^{* *}$ & .040 \\
\hline \multicolumn{8}{|l|}{ Responsibility for persons } \\
\hline Job dissatisfaction & $-0.158^{* *}$ & -0.040 & & & & $.067^{* *}$ & .064 \\
\hline Boredom & $-0.276^{* *}$ & 0.070 & & & & $.102^{* * *}$ & .099 \\
\hline \multicolumn{8}{|l|}{ Quantitative work load } \\
\hline Job dissatisfaction & 0.118 & $-0.508^{* *}$ & & & & $.072^{* *}$ & .069 \\
\hline Work-load dissatisfaction & $0.377^{* *}$ & $-0.455^{* *}$ & $0.402^{* *}$ & $-0.40 \mathrm{I}^{*}$ & -0.110 & $.322^{* *}$ & .316 \\
\hline Boredom & $-0.159^{*}$ & $-0.605^{* *}$ & & & & $.078^{* *}$ & .075 \\
\hline Depression & $0.110^{* *}$ & $-0.266^{* *}$ & & & & $.060^{* * *}$ & .057 \\
\hline Irritation & $0.231^{* *}$ & $-0.137^{* *}$ & & & & $.066^{* *}$ & .063 \\
\hline
\end{tabular}

Note. Ns ranged from 615 to 634 . Values for environment $(E)$, person $(P), E^{2}, E P$, and $P^{2}$ are unstandardized regression coefficients for equations in which all predictors were entered simultaneously. $R^{2}$ is the squared multiple correlation coefficient, and $R_{\mathrm{adj}}^{2}$ is the adjusted squared multiple correlation coefficient. Probability levels for the $R^{2}$ s were corrected using the sequential Bonferroni procedure described by Holm (1979), assuming a total of 28 tests; all remained significant at $p<.001$.

$* p<.05 . * * p<.01$.

along the $E=P$ line, with the sign indicated by $a_{1}$. If $a_{2}$ is positive, then the surface is curved upward (i.e., convex) along the $E=P$ line, whereas if $a_{2}$ is negative, the surface is curved downward (i.e., concave) along the $E=P$ line. (In either case, $a_{1}$ indicates the slope where $E=0$, which represents the midpoint of the $E, P$ plane when data are scale centered) Tests of $a_{1}$ and $a_{2}$ are conducted by dividing each by its standard error, which is calculated using standard rules for determining the variance of a sum of random variables. Alternately, $a_{1}$ and $a_{2}$ can be tested individually or jointly by determining whether their corresponding coefficient sums differ from 0 , using the MGLH module of SYSTAT. Note that, because $E=P$ in Equation $14, P$ can be substituted for $E$ with no effect on the obtained results.

Next, consider lateral shifts in the surface along the $E=-P$ line, perpendicular to the $E=P$ line. The magnitude and direction of the shift along the $E=-P$ line is given by the quantity $\left(b_{2}-b_{1}\right) / 2\left(b_{3}-b_{4}+b_{5}\right)$. A positive value indicates a shift toward the region where $E>P$, whereas a negative value indicates a shift toward the region where $E<P$. Note that, if the fit squared constraints hold, $b_{1}$ and $b_{2}$ are both 0 and the surface remains centered along the $E=P$ line, as in Figure $1 \mathrm{j}$. If $b_{1}$ and $b_{2}$ are equal in both magnitude and sign, the surface again remains centered along the $E=P$ line, although its slope at the midpoint of that line (i.e., $a_{1}$ ) is no longer 0 .

Finally, consider rotations in the surface, such that its minimum no longer lies along the $E=P$ line. The magnitude and direction of these rotations are a function of $b_{3}, b_{4}$, and $b_{5}$. If $b_{3}$ and $b_{5}$ are equal, then the surface does not rotate, independent of $b_{4}$. If $b_{3}$ is less than $b_{5}$, the surface rotates clockwise, whereas if $b_{3}$ is greater than $b_{5}$, the surface rotates counterclockwise. In either case, the magnitude of the rotation is determined not only by the difference between $b_{3}$ and $b_{5}$ but also by $b_{4}$, with larger rotations for smaller values of $b_{4}$. Note that rotations can occur in combination with lateral shifts, such that the minimum of the surface is not only displaced laterally from the $E=$ $P$ line but is also no longer parallel to that line. Also note that, in addition to indicating rotations in the surface, $b_{3}, b_{4}$, and $b_{5}$ influence the curvature of the surface along the $E=P$ line through their effect on $a_{2}$, as described earlier.

These principles were first applied to the equation for job complexity predicting boredom, which yielded positive coefficients on $E^{2}$ and $P^{2}$ and negative coefficients on $E$ and $E P$. The joint test of $a_{1}$ and $a_{2}$ was significant, $F(2,611)=62.03, p<.01$, as were the individual tests of $a_{1}, F(1,611)=78.08, p<.01$, and of $a_{2}, F(1,611)=6.18, p<.05$. The signs on $a_{1}$ and $a_{2}$ were negative and positive, respectively, indicating a steep negative slope along the $E=P$ line, with a slight upward curvature. The quantity $\left(b_{2}-b_{1}\right) / 2\left(b_{3}-b_{4}+b_{5}\right)$ was 0.459 , indicating a shift toward the region where $E>P$ of about half a unit along the $E=$ $-P$ line. Furthermore, $b_{3}$ was slightly larger than $b_{5}$, indicating a modest counterclockwise rotation. In combination, these results indicated a surface much like that depicted in Figure 2i, where boredom was lower when job complexity/ $E$ and job complexity/ $P$ were both high than when both were low and, for a given level of $P$, where boredom was minimized when $E$ slightly exceeded $P$ rather than when $E$ and $P$ were equal, particularly at low levels of $E$ and $P$.

The equations for job complexity predicting job dissatisfaction and work-load dissatisfaction yielded positive coefficients on $E^{2}$ and negative coefficients on $P$ and $E P$. In both cases, $a_{1}$ 
was negative and significant $(p<.05)$ and $a_{2}$ was not significant, thereby indicating a negative linear slope along the $E=P$ line. The quantity $\left(b_{2}-b_{1}\right) / 2\left(b_{3}-b_{4}+b_{5}\right)$ was also small and negative in both cases $(-0.042$ for job dissatisfaction and -0.371 for work-load dissatisfaction), indicating a small shift toward the region where $E<P$. In addition, $b_{3}$ was larger than $b_{5}$, particularly for work-load dissatisfaction, indicating a counterclockwise rotation. Taken together, these results indicated two major deviations from the surface depicted in Figure lj. First, as with boredom, job dissatisfaction and work-load dissatisfaction were lower when job complexity $/ E$ and job complexity/ $P$ were both high than when both were low. Second, when $E$ and $P$ were both low, dissatisfaction was minimized when $E$ was slightly greater than $P$, but when $E$ and $P$ were both high, dissatisfaction was minimized when $E$ was slightly less than $P$.

The equation for job complexity predicting depression yielded a positive coefficient on $E^{2}$ and a negative coefficient on $E P$. However, $a_{1}$ and $a_{2}$ were not significant either individually or jointly, indicating that the surface was essentially flat along the $E=P$ line. The quantity $\left(b_{2}-b_{1}\right) / 2\left(b_{3}-b_{4}+b_{5}\right)$ was -0.148 , and $b_{3}$ was notably larger than $b_{5}$, indicating a slight shift toward the region where $E<P$ along with a counterclockwise rotation. These results indicated a surface much like Figure lj except for a counterclockwise rotation, such that when $E$ and $P$ were both low, depression was lowest when $E$ was slightly greater than $P$, but when $E$ and $P$ were both high, depression was lowest when $E$ was slightly less than $P$.

For job complexity predicting anxiety, the coefficients on $E$ and $E^{2}$ were positive, whereas the coefficient on $E P$ was negative. The joint test of $a_{1}$ and $a_{2}$ was significant, $F(2,613)=5.85$, $p<.01$, as were individual tests for $a_{1}, F(1,613)=6.07, p<.01$, and for $a_{2}, F(1,613)=11.63, p<.01$. The signs on $a_{1}$ and $a_{2}$ were positive and negative, respectively, indicating that the surface was positively sloped but concave along the $E=P$ line. The quantity $\left(b_{2}-b_{1}\right) / 2\left(b_{3}-b_{4}+b_{5}\right)$ was -0.216 , and $b_{3}$ and $b_{5}$ were essentially equal, indicating a slight shift toward the region where $E<P$ with no appreciable rotation. Taken together, these results indicated that anxiety was lowest when job complexity/ $E$ was slightly less than job complexity $/ P$ and increased in either direction and also that anxiety was higher when $E$ and $P$ were both moderate than when they were both either high or low.

Finally, the equation for quantitative work load predicting work-load dissatisfaction yielded positive coefficients on $E$ and $E^{2}$ and negative coefficients on $P$ and $E P$. Individual and joint tests of $a_{1}$ and $a_{2}$ were not significant, indicating that the surface was essentially flat along the $E=P$ line. The quantity $\left(b_{2}-\right.$ $\left.b_{1}\right) / 2\left(b_{3}-b_{4}+b_{5}\right)$ was -0.600 , and $b_{3}$ was notably larger than $b_{5}$, indicating a moderate shift toward the region where $E<P$ and a substantial counterclockwise rotation. These results indicated a surface similar to Figure $3 \mathrm{i}$, in which, when quantitative work $\operatorname{load} / E$ and quantitative work load/ $P$ were both high, work-load dissatisfaction was lowest when $E$ was notably less than $P$, but when $E$ and $P$ were both low, work-load dissatisfaction was lowest when $E$ was slightly greater than $P$.

\section{Summary of Results}

The results of this study provided little support for the restricted models depicted by the fit, deficiency, excess, poor fit, and fit squared measures used by French et al. (1982). Although these measures collectively yielded 45 significant correlations with strain, the constraints imposed by these measures were supported in only three instances. Relaxing these constraints increased adjusted squared multiple correlations from an average of .024 for the constrained equations to .059 for the unconstrained equations-more than doubling the proportion of explained variance. Exploratory analyses identified four general classes of models. One class involved significant relationships for either $E$ or $P$ and, hence, provided no evidence for fit. A second class indicated relationships of the same sign for $E$ and $P$ that, although theoretically plausible, are not accounted for by P-E fit theory. A third class involved opposite relationships for $E$ and $P$, corresponding to the fit model. Finally, the fourth class consisted of various modifications of the fit squared model, with slope or curvilinearity along the $E=P$ line, shifts or rotations of the region of minimum strain from the $E=P$ line, or some combination thereof. Taken together, these models bear certain basic similarities to those underlying the fit measures used by French et al., particularly the models for fit and fit squared measures, but also indicate substantively meaningful relationships that these measures cannot adequately represent.

The results of this study resolved ambiguities in the findings reported by French et al. (1982), thereby allowing firmer conclusions regarding the relationship between $E, P$, and strain. In some cases, our conclusions supported those drawn by French et al., with certain modifications. For example, French et al. concluded that work-load dissatisfaction increased as work$\operatorname{load} / E$ deviated from work-load/P, particularly when $E$ exceeded $P$. The present study supported this conclusion but added that, at low levels of $E$ and $P$, work-load dissatisfaction was lowest when $E$ slightly exceeded $P$, whereas at high levels of $E$ and $P$, work-load dissatisfaction was lowest when $E$ was notably less than $P$.

French et al. (1982) further concluded that job dissatisfaction, work-load dissatisfaction, boredom, depression, and anxiety increased when job complexity/ $E$ deviated from job complexity/P. They also noted several asymmetries, with a stronger relationship for boredom when $E$ fell short of $P$ and stronger relationships for work-load dissatisfaction, depression, and anxiety when $E$ exceeded $P$. The present study supported these conclusions, with four modifications. First, job dissatisfaction, work-load dissatisfaction, and boredom were higher when job complexity $/ E$ and job complexity $/ P$ were both low than when both were high. Second, when job complexity/E and job complexity/P were both low, job dissatisfaction, work-load dissatisfaction, and depression were lowest when $E$ was slightly greater than $P$, whereas when $E$ and $P$ were both high, these strains were lowest when $E$ was slightly less than $P$. Third, for all levels of job complexity $/ E$ and job complexity $/ P$, boredom was lower when $E$ slightly exceeded $P$ than when $E$ and $P$ were equal, particularly when $E$ and $P$ were both low. Finally, anxiety was higher when job complexity/ $E$ and job complexity $/ P$ were both moderate than when they were both high or low.

Although many features of the three-dimensional relationship between $E, P$, and strain were not detected by French et al. (1982), these features are, for the most part, consistent with P-E fit theory (Caplan, 1987; French et al., 1982; Harrison, 1978, 
1985). For example, Harrison (1978) noted that strain may be minimized at points other than perfect fit. We found this for job complexity, for which a slight excess was often associated with lower strain when $E$ and $P$ were both low. This seems reasonable, given that perfect fit for very simple jobs may result in monotony and, hence, strain. Conversely, at high levels of job complexity $/ E$ and job complexity $/ P$, strain was often lowest when $E$ was slightly less than $P$. This suggests that maintaining fit for complex jobs may be taxing and that conceding to a slight deficiency may result in lower overall strain. A similar interpretation applies to the relationship between quantitative work load and work-load dissatisfaction, in which dissatisfaction was lower for a modest deficiency than for perfect fit when $E$ and $P$ were both high.

$P$-E fit theory also indicates that perfect fit may yield different levels of strain, depending on the absolute levels of $E$ and $P$ (French et al., 1982; Harrison, 1978; see also Imparato, 1972). We found this for job complexity predicting job dissatisfaction, work-load dissatisfaction, and boredom, which were lower when $E$ and $P$ were both high than when both were low. This may represent the effects of fit on other job dimensions, in that complex jobs typically entail high pay, status, recognition, and other valued rewards (Harrison, 1978). Alternatively, this may reflect the operation of two standards when actual job complexity is evaluated: one representing the personal preferences of the employee, and another reflecting workplace, professional, or societal norms that place value on complex jobs. Anxiety also varied along the line of perfect fit for job complexity, with greater anxiety when $E$ and $P$ were both moderate. This may indicate that achieving fit at moderate levels of job complexity $/ E$ and job complexity $/ P$ involves greater uncertainty than does achieving fit at high or low levels of $E$ and $P$, resulting in increased anxiety (cf. Beehr \& Bhagat, 1985; McGrath, 1976). Future research into P-E fit theory should include measures necessary to substantiate explanations such as these.

Our resuits also indicated that certain conclusions drawn by French et al. (1982) should be revised. For example, French et al. reported that job dissatisfaction, work-load dissatisfaction, boredom, and depression increased when role ambiguity/ $E$ deviated from role ambiguity $/ P$, with stronger relationships for excess ambiguity. In contrast, we found that job dissatisfaction, work-load dissatisfaction, and depression were essentially positive monotonic functions of role ambiguity/ $E$. We also found that, with the sequential Bonferroni correction, many of the correlations for role ambiguity discussed by French et al., including those for boredom, were no longer significant.

French et al. (1982) also concluded that either too much or too little responsibility for persons was related to increased dissatisfaction and boredom, particularly when responsibility for persons was insufficient. In contrast, we found that job dissatisfaction and boredom were simply negatively related to responsibility for persons $/ E$. French et al. also reported that excess responsibility for persons was associated with increased work-load dissatisfaction and anxiety. Using the first random subsample, we obtained results that supported the findings of French et al. for work-load dissatisfaction, but when we conducted exploratory analyses using both subsamples, neither of these findings were supported.

Finally, French et al. (1982) reported that when quantitative work load $/ E$ deviated from quantitative work load $/ P$, job dissatisfaction, depression, and irritation increased, particularly when quantitative work load was excessive. In contrast, we found that these strains were positively related to quantitative work load $/ E$ and negatively related to quantitative work load $/ P$, analogous to the fit model. French et al. also reported that boredom increased symmetrically as quantitative work load/ $E$ deviated from quantitative work load $/ P$, whereas we found that boredom was negatively related to both $E$ and $P$ measures of quantitative work load.

\section{Limitations}

Although the present study has substantially clarified and refined the results reported by French et al. (1982), several limitations should be noted. Many of these limitations, such as using only male respondents, collapsing across heterogeneous occupations, relying on cross-sectional data, the potential confounding of $E$ and $P$ measures with fit, and bias resulting from measurement error, were discussed in the original reports $(\mathrm{Ca}$ plan et al., 1980; French et al., 1982) and, hence, are not repeated here. However, the present study contained some additional limitations. For example, to maintain manageable size and scope, we omitted four job dimensions and 11 indexes of physiological and behavioral strain that were examined by French et al. Consequently, all of our analyses were based on self-report measures, which may have spuriously inflated the observed relationships. In addition, to avoid bias resulting from unequal occupational representation, we used only about one third of the available data. It would be useful to conduct followup analyses of occupational differences using the full sample, such as those conducted by French et al. Furthermore, the two subsamples used for our exploratory analyses and cross-validations were both drawn from the full sample of 2,010 respondents rather than from the larger population of employed adults, thereby limiting generalizability (Murphy, 1983). Finally, certain aspects of the three-dimensional surfaces yielded by the exploratory analyses, such as shifts and rotations of the region of minimum strain, were described but not formally tested. This was due to the mathematical expressions for surface shifts and rotations, which involve products, ratios, and powers of the coefficient estimates from the quadratic equation and, hence, are not amenable to conventional tests of statistical significance. Future research should attempt to provide more objective criteria for describing and testing the exploratory surfaces yielded by the Edwards (in press) procedure (for one approach, see Edwards \& Parry, in press).

\section{Implications for P-E Fit Research}

This study has several implications for P-E fit research. First, fit measures that collapse $E$ and $P$ into a single score should be abandoned in favor of polynomial equations containing $E, P$, and appropriate higher order terms (Edwards, 1991, in press; Edwards \& Cooper, 1990; Edwards \& Parry, in press). As this study demonstrated, fit measures yield ambiguous results, confound the separate relationships of $E$ and $P$ with strain, impose a highly restrictive set of constraints that are rarely supported, and reduce the inherently three-dimensional relationship be- 
tween $E, P$, and strain to two dimensions. The Edwards (in press) procedure avoided these problems and showed that in most cases, the relationship between $E, P$, and strain could not be adequately represented by the fit measures used by French et al. (1982). Because this procedure uses equations that subsume fit measures, it eliminates the need for these measures and, furthermore, allows tests of a much wider range of surfaces relating $E$ and $P$ to strain.

A second, related implication is that tests of fit should not focus on the increment in variance explained by fit measures beyond their components, as is often done in P-E fit research. These tests are flawed, not only because they rely on fit measures but also because they do not support the model in question unless the coefficients on $E$ and $P$ happen to be zero, which is rarely verified. In any case, the fundamental question in P-E fit research is not whether a given fit measure explains variance beyond $E$ and $P$, but whether the joint relationships of $E$ and $P$ with strain follow the functional form corresponding to the conceptual model of interest. These relationships can be readily tested using appropriate polynomial regression equations, thereby avoiding the need for tests of fit measures, either independently or after controlling for $E$ and $P$.

Third, hypotheses regarding bivariate relationships between fit measures and strain should be recast in terms of the direction and relative magnitude of coefficients on $E, P$, and appropriate higher order terms in polynomial regression equations. For example, hypothesizing a negative relationship between fit and strain is logically equivalent to hypothesizing a negative coefficient on $E$ and a positive coefficient on $P$ (see Figures 1a and 1 b). Similarly, a positive relationship for fit squared implies positive coefficients on $E^{2}$ and $P^{2}$, a negative coefficient on $E P$, and nonsignificant coefficients on $E$ and $P$ along the $E=P$ line (Figures $\mathrm{li}$ and $\mathrm{lj}$ ). As stated, these hypotheses correspond to weak tests of the fit and fit squared models, because they merely specify the direction of the coefficients. Strong tests would add hypotheses regarding relative coefficient magnitudes, as represented by the constraints imposed by the fit and fit squared measures. By stating hypotheses in these terms, researchers can consider evidence for both strong and weak versions of fit models, which allows more complete tests of these models and avoids reductions in explained variance caused by routinely imposing constraints that may be more restrictive than required by the model of interest.

Fourth, specific hypotheses should be developed for coeffcient patterns representing models more complex than the fit, deficiency, excess, poor fit, and fit squared models. Hypotheses for these five models are embodied in their corresponding fit measures and, hence, are fairly easy to derive. However, a major advantage of the Edwards (in press) procedure is that it allows tests of models that are more complex than models underlying fit measures. Hypotheses for some of these models can be derived from the coefficients reported in Table 6 . For example, a $U$-shaped surface with a negative slope along the $E=P$ line is represented by coefficients on $E^{2}, E P$, and $P^{2}$ corresponding to the fit squared model, supplemented by negative coefficients on $E$ and $P$. Similarly, a $U$-shaped surface with a clockwise rotation off the $E=P$ line is represented by reductions in the absolute magnitudes of the coefficients on $E^{2}$ and $E P$ relative to the pattern predicted by the fit squared model, and a U-shaped surface with a counterclockwise rotation is represented by reductions in the absolute magnitudes of the coefficients on $E P$ and $P^{2}$. A $U$-shaped surface with its minimum parallel to the $E=P$ line but shifted toward the $E>P$ region implies a negative coefficient on $E$ and a positive coefficient on $P$, whereas a surface shifted toward the $E<P$ region implies a positive coeffcient on $E$ and a negative coefficient on $P$. Hypotheses regarding other surfaces may be derived by determining the magnitude and direction of the coefficients in their corresponding polynomial equations (Edwards \& Parry, in press).

Finally, equations derived through exploratory analyses should be cross-validated whenever possible. Some equations obtained in the preceding exploratory analyses contained cubic and quartic terms. Although these terms allowed rather precise description of the data, they represented relationships that defy meaningful interpretation, given current theory. Fortunately, none of these terms survived cross-validation, suggesting that they merely represented sampling variability. Without cross-validation, these terms would have demanded rather elaborate theoretical explanations that probably would have failed to generalize beyond the data at hand.

\section{Practical Implications}

This study suggests several practical implications that go beyond traditional guidelines for maximizing P-E fit (e.g., Dawis \& Lofquist, 1984; French et al., 1982; Harrison, 1978; J. L. Holland, 1985; Schein, 1978). First, even when perfect fit is attained, strain may be further reduced by changing the absolute levels of $E$ and $P$. This was evidenced by surfaces relating job complexity to job dissatisfaction, work-load dissatisfaction, and boredom, which showed that strain was lower when both $E$ and $P$ were high than when both were low. Second, strain may be decreased by creating modest levels of misfit, again depending on the absolute levels of $E$ and $P$. This was shown by surfaces for job complexity that were shifted and rotated off the $E=P$ diagonal. Third, for some job dimensions, strain may be decreased by changes in $E$ and $P$ without regard to fit. This was implied by results for role ambiguity, which showed that strain was essentially a positive monotonic function of actual role ambiguity. Of course, these recommendations are tentative, given that they are based on a single, cross-sectional study. Nonetheless, they suggest that interventions can be more specific and, hence, more effective when the three-dimensional relationship between $E, P$, and strain is considered.

\section{Conclusion}

This study has substantially clarified and refined the results reported by French et al. (1982) regarding the relationship between P-E fit and strain. The French et al. results were often ambiguous, because of the use of fit measures that collapsed $E$ and $P$ into a single score. The present study used a procedure described by Edwards (in press) that avoids the problems with fit measures and preserves the inherently three-dimensional relationship between $E, P$, and strain. This procedure resolved ambiguities in the original results and showed that the relationship between $E, P$, and strain was often more complex than could be depicted by the fit measures used by French et al. By 
using this procedure in future P-E fit research, researchers may conduct more complete and precise tests of the three-dimensional relationship between $E, P$, and strain and explore the full range of predictions offered by P-E fit theory.

\section{References}

Aiken, L. A., \& West, S. G. (1991). Multiple regression: Testing and interpreting interactions. Newbury Park, CA: Sage.

Beehr, T. A., \& Bhagat, R. S. (1985). Introduction to human stress and cognition in organizations. In T. A. Beehr \& R. S. Bhagat (Eds.), Human stress and cognition in organizations (pp. 3-19). New York: Wiley.

Box, G. E. P., \& Draper, N. R. (1987). Empirical model-building and response surfaces. New York: Wiley.

Caplan, R. D. (1987). Person-environment fit theory and organizations: Commensurate dimensions, time perspectives, and mechanisms. Journal of Vocational Behavior, 31, 248-267.

Caplan, R. D., Cobb, S., French, J. R. P., Jr., Harrison, R. V., \& Pinneau, S. R. (1980). Job demands and worker health: Main effects and occupational differences. Ann Arbor, MI: Institute for Social Research.

Cohen, J. (1988). Statistical power analysis for the behavioral sciences (2nd ed.). San Diego, CA: Academic Press.

Cohen, J., \& Cohen, P. (1983). Applied multiple regression/correlation analysis for the behavioral sciences (2nd ed.). Hillsdale, NJ: Erlbaum.

Cronbach, L. J. (1987). Statistical tests for moderator variables: Flaws in analyses recently proposed. Psychological Bulletin, 102, 414-417.

Cronbach, L. J., \& Furby, L. (1970). How we should measure "change" -Or should we? Psychological Bulletin, 74, 68-80.

Cronbach, L. J., \& Gleser, G. C. (1953). Assessing the similarity between profiles. Psychological Bulletin, 50, 456-473.

Darlington, R. B. (1990). Regression and linear models. New York: McGraw-Hill.

Dawis, R. V., \& Lofquist, L. H. (1984). A psychological theory of work adjustment. Minneapolis: University of Minnesota Press.

Edwards, J. R. (1991). Person-job fit: A conceptual integration, literature review, and methodological critique. In C. L. Cooper \& I. T. Robertson (Eds.), International review of industrial and organizational psychology (Vol. 6, pp. 283-357). New York: Wiley.

Edwards, J. R. (1992). A cybernetic theory of stress, coping, and wellbeing in organizations. Academy of Management Review, 17, 238274.

Edwards, J. R. (in press). The study of congruence in organizational behavior research: Critique and a proposed alternative. Organizational Behavior and Human Decision Processes.

Edwards, J. R., \& Cooper, C. L. (1990). The person-environment fit approach to stress: Recurring problems and some suggested solutions. Journal of Organizational Behavior, 10, 293-307.

Edwards, J. R., \& Parry, M. E. (in press). On the use of polynomial regression equations as an alternative to difference scores in organizational research. Academy of Management Journal.

Eulberg, J. R., Weekley, J. A., \& Bhagat, R. S. (1988). Models of stress in organizational research: A metatheoretical perspective. Human Relations, 41, 331-350.

French, J. R. P., Jr., Caplan, R. D., \& Harrison, R. V. (1982). The mechanisms of job stress and strain. New York: Wiley.

Harrison, R. V. (1978). Person-environment fit and job stress. In C. L.
Cooper \& R. Payne (Eds.), Stress at work (pp. 175-205). New York: Wiley.

Harrison, R. V. (1985). The person-environment fit model and the study of job stress. In T. A. Beehr \& R. S. Bhagat (Eds.), Human stress and cognition in organizations (pp. 23-55). New York: Wiley.

Hochberg, Y., \& Tamhane, A. C. (1987). Multiple comparison procedures. New York: Wiley.

Holland, B. S., \& Copenhaver, M. D. (1988). Improved Bonferroni-type multiple testing procedures. Psychological Bulletin, 104, 145-149.

Holland, J. L. (1985). Making vocational choices (2nd ed.). Englewood Cliffs, NJ: Prentice Hall.

Holm, S. (1979). A simple sequentially rejective multiple test procedure. Scandinavian Journal of Statistics, 6, 65-70.

Imparato, N. (1972). Relationship between Porter's Need Satisfaction Questionnaire and the Job Descriptive Index. Journal of Applied Psychology, 56, 397-405.

Jaccard, J., Turrisi, R., \& Wan, C. K. (1990). Interaction effects in multiple regression. Newbury Park, CA: Sage.

Johns, G. (1981). Difference score measures of organizational behavior variables: A critique. Organizational Behavior and Human Performance, 27, 443-463.

Karasek, R. A., Jr. (1979). Job demands, job decision latitude, and mental strain: Implications for job redesign. Administrative Science Quarterly, 24, 285-308.

Khuri, A. I., \& Cornell, J. A. (1987). Response surfaces: Designs and analyses. New York: Marcel Dekker.

McGrath, J. E. (1976). Stress and behavior in organizations. In M. D. Dunnette (Ed.), Handbook of industrial and organizational psychology (pp. 1351-1395). Chicago: Rand McNally.

McLain, D. H. (1974). Drawing contours from arbitrary data points. The Computer Journal, 17, 318-324.

Miller, R. G., Jr. (1981). Simultaneous statistical inference (2nd ed.). New York: Springer-Verlag.

Mosier, C. I. (1951). Symposium: The need and means of cross-validation. Educational and Psychological Measurement, 11, 5-11.

Murphy, K. R. (1983). Fooling yourself with cross-validation: Single sample designs. Personnel Psychology, 36,111-118.

Neter, J., Wasserman, W., \& Kutner, M. H. (1989). Applied linear regression models (2nd ed.). Homewood, IL: Irwin.

Schein, E. (1978). Career dynamics: Matching individual and organizational needs. Reading, MA: Addison-Wesley.

Schuler, R. S. (1980). Definition and conceptualization of stress in organizations. Organizational Behavior and Human Performance, $25,184-215$.

Seaman, M. A., Levin, J. R., \& Serlin, R. C. (1991). New developments in pairwise multiple comparisons: Some powerful and practicable procedures. Psychological Bulletin, 110, 577-586.

Wall, T. D., \& Payne, R. (1973). Are deficiency scores deficient? Journal of Applied Psychology, 58, 322-326.

Werts, C. E., \& Linn, R. L. (1970). A general linear model for studying growth. Psychological Bulletin, 73, 17-22.

Wilkinson, L. (1990a). SYGRAPH: The system for graphics. Evanston, IL: SYSTAT.

Wilkinson, L. (1990b). SYSTAT: The system for statistics. Evanston, IL: SYSTAT.

Received February 27, 1992

Revision received October 15, 1992

Accepted November 6,1992 